\title{
Artefacts urbanistiques en périphérie de Rio de Janeiro: la technologie du lotissement
}

\author{
Thomas Cortado' \\ 'Universidade Federal do Rio de Janeiro, Museu Nacional, Programa de Pós-graduação em \\ Antropologia Social - PPGAS, Rio de Janeiro, Brasil
}

\section{Résumé}

L’agencement spatial des périphéries urbaines, au travers du « lotissement » (loteamento), n’a pas suscité beaucoup d'intérêt de la part des anthropologues. Prenant appui sur les documents d'urbanisme impliqués dans les opérations de lotissement, cet article prétend faire la " généalogie " (Foucault) de cette forme d'habitat à Rio de Janeiro en analysant les différentes rationalités techniques et politiques qui la traversent. En particulier, il défend que le lotissement doit beaucoup à la " raison graphique » (Goody), au genre d'opérations que l'utilisation « d'artefacts graphiques » (Hull) rend possible. Il soutient également que la technologie du lotissement est née en réponse à des problèmes spécifiques: à l'époque coloniale, il s'agissait de réglementer la création de voirie pour préserver l'espace public de la rue, alors qu'à la fin du dix-huitième siècle, l'enjeu était plutôt de réformer le réseau des voies de circulation. La codification des opérations de lotissement telles que nous les connaissons aujourd'hui ne survint qu'au début du siècle précédent en accord avec les nouvelles préoccupations de l'époque concernant les nécessités collectives de la population urbaine. Loin des stéréotypes sur la " ville informelle " (cidade ilegal), la périphérie se révèle un véritable laboratoire de l’aménagement urbain (planejamento urbano).

Mots-clés: Périphérie, Lotissement, Aménagement Urbain, Documents, Gouvernementalité. 


\section{Artefatos urbanísticos na periferia do Rio de Janeiro:}

\section{a tecnologia do loteamento}

\section{Resumo}

O ordenamento espacial das periferias, através do loteamento, não despertou tanto interesse entre os antropólogos. Com base em pesquisa sobre documentos, principalmente planos urbanísticos, e editais de leis, pretendemos fazer uma "genealogia" (Foucault) do loteamento, analisando as várias racionalidades, técnicas e políticas, que contribuíram para esta forma de morar. Argumentamos que o loteamento deve muito à "razão gráfica" (Goody), ao tipo de operações que o uso de "artefatos gráficos" (Hull) possibilita. Defendemos também que esta tecnologia responde a problemas específicos: no período colonial, regulamentava-se a abertura de ruas para defender os espaços públicos, enquanto, no final do século dezoito, o problema já era de reformar a malha viária. A codificação do loteamento tal como conhecemos só ocorreu no início do século vinte, em decorrência de novas problemáticas acerca das necessidades coletivas da população urbana. Longe de constituir uma cidade ilegal, a periferia aparece assim como um laboratório do planejamento urbano.

Palavras-chaves: Periferia, Loteamento, Planejamento Urbano, Documentos, Governamentalidade. 


\title{
Artefacts urbanistiques en périphérie de Rio de Janeiro:
}

\section{la technologie du lotissement}

\begin{abstract}
The spatial ordering of Rio de Janeiro's poor suburbs, through subdivision (loteamento), did not raise such interest among anthropologists. Based on documents, mostly urban plans, and legislation, we intend to make a "genealogy" (Foucault) of the subdivision, giving attention to technical and political rationalities. We argue that the subdivision owes much to the "graphic reason" (Goody), to the kind of operations that the use of "graphic artifacts" allows. Moreover, this technology responds to specific problems: during colonial times, government regulated street opening in order to defend public spaces. At the end of the eighteenth century, the problem was how to reform the road network. Current subdivision regulations come from the early twentieth century, when new issues about collective needs aroused. Far from being an illegal city, poor suburbs appear as a laboratory for urban planning.
\end{abstract}

Keywords: Urban Periphery, Subdivision, Urban Planning, Documents, Governmentality. 


\title{
Artefacts urbanistiques en périphérie de Rio de Janeiro:
}

\section{la technologie du lotissement}

\author{
Thomas Cortado
}

\section{Analytique de la périphérie: le lotissement comme objet ${ }^{1}$}

Dans son célèbre article « Vieilles nouveautés dans les modes d'urbanisation brésiliens », Carlos Nelson Ferreira s'en prenait aux approches qui réduisaient « la localisation des personnes dans l'espace urbain et dans leurs formes d'habitat aux déterminations englobantes et un tantinet abstraites de la domination de l'État et du Capital " (Santos 1980: 18). Loin de vouloir invalider la vision marxiste, l'architecte et anthropologue carioca soulignait d'abord " la complexité des articulations » en vigueur dans le processus d'urbanisation. En particulier dans la " périphérie urbaine " (periferia)', cette frange pauvre et éloignée de l'espace métropolitain, dépourvue d'infrastructures, où prédominent " l'autoconstruction » (autoconstrução) et la petite propriétés, « l'ensemble des actions que l'on désigne par HABITER dans une ville » mobilise un large éventail d'acteurs, tels que les propriétaires ruraux, les compagnies immobilières, les services municipaux, les notaires (cartórios), les correcteurs immobiliers, les réseaux familiaux et de connaissances, les politiciens et leurs clientèles, etc. De leur action commune, mais pas forcément coordonnée, surgissaient des quartiers socialement hétérogènes, plus ou moins formalisés, que la plupart des spécialistes situaient sur un continuum allant de la périphérie la plus " proche » du centre, la mieux équipée en services de consommation collective, à la périphérie la plus " distante ", dénuée d’à peu près tout (Santos 1977; Abreu 2013). "Le fait d'habiter en Périphérie ", analysait Santos, " obéit à une série d'articulations entre divers acteurs et leurs processus qui viabilisèrent la production, la commercialisation, l'accès et la consommation du logement dans n'importe laquelle des instances représentatives (économique, juridique, financière, politique, socioculturelle, technologique, etc.), à travers la solution des LOTISSEMENTS » (Santos 1980: 28). C'était vers le lotissement, autrement dit vers l'opération (ou le résultat de l'opération) qui consiste à simultanément créer une nouvelle voirie et à diviser une unité foncière en propriétés ${ }^{4}$, que convergeait l'ensemble des acteurs: le propriétaire rural mettait à disposition un terrain autrefois réservé à un usage agricole (ou alors était la victime d'usurpateurs, les grileiros), la compagnie se

\footnotetext{
1 Le présent article est un prolongement de ma thèse de doctorat qui porte sur l'urbanisation du Jardim Maravilha, lotissement situé en périphérie de Rio de Janeiro (Cortado 2018). Je remercie la Coordination pour le Perfectionnement du Personnel de Niveau Supérieur (CAPES) pour son soutien financier ainsi que le séminaire des élèves du Programme de Troisième Cycle en Anthropologie Sociale (PPGAS) où j’ai pu présenter une première version de ce texte. Je tiens aussi à remercier Lucie Elgoyhen pour la révision et correction de cet article et les coordonnateurs de ce numéro, en particulier Jussara Freire, pour leurs précieuses suggestions.

2 Dans la suite de cet article, nous réserverons l'usage de l'italique aux catégories de la langue brésilienne et du vocabulaire indigène, en plus des titres d'ouvrage ou des mots étrangers. On emploiera les guillemets dans la traduction de ces mêmes catégories ainsi que pour les citations et concepts empruntés à la littérature.

3 Ce triptyque " périphérie-autoconstruction-lotissement " a dominé les travaux des années soixante-dix et quatre-vingt sur le thème de la périphérie (Valladares \& Figueiredo 1981: 35). La périphérie se trouvait alors au centre d'importantes discussions théoriques, d’inspiration marxiste, bien représentées dans le fameux ouvrage collectif édité par Ermínia Maricato, La production capitaliste de la maison (et de la ville) dans le Brésil industriel (1979). Cette tradition de recherche a connu un certain déclin depuis la fin des années quatre-vingt, si l'on excepte les travaux de Rolnik sur la législation urbaine (2005) et ceux de Bonduki sur la politique du logement (1999), le mot même de périphérie devenant un vague synonyme de marginalité sociale et culturelle.

4 Il est d'usage dans le droit brésilien, depuis les années soixante au moins, de distinguer entre le « démembrement " (desmembramento), l'opération qui consiste à seulement diviser un terrain en parcelles, et le lotissement proprement dit, lorsque la division foncière, parce qu'elle concerne une vaste région, oblige à la création d'une voirie. Démembrement et lotissement font partie de ce que le droit administratif appelle le " parcellement du sol » (parcelamento do solo).
} 
chargeait des travaux de lotissement (ne respectant pas toujours la législation), la municipalité approuvait le projet (ou n'avait même pas vent de l'opération), le notaire authentifiait (ou non) les transactions, les correcteurs immobiliers prenaient en charge la vente des parcelles (lotes), etc., jusqu'à l'habitant, qui recrutait ses amis et ses proches pour l'aider non seulement à construire sa maison, mais aussi à urbaniser le lotissement 5 .

Hybrides, les « lotissements défectueux » (loteamentos irregulares) ${ }^{6}$ de la périphérie ne sont ni le fait du gouvernement, à l'image des " cités " (conjuntos habitacionais) dont l'existence à Rio de Janeiro remonte aux années trente, ni le produit d'une croissance dite " organique ", comme ce serait le cas des favelas ; ils occupent en fait une région ambiguë dans les systèmes de classifications scientifiques et indigènes de l'espace urbain, quelque part entre les catégories de " ville informelle " et de " ville formelle ", ce qui certainement explique leur moindre visibilité dans l'espace public. De ce fait, cet habitat intéresse peu les chercheurs, ce que Santos regrettait déjà au début des années quatre-vingt, quoiqu'il n'y eût jamais autant de travaux sur les lotissements, en particulier sur les lotissements cariocas, qu'à cette époque. Ainsi, dans les « lotissements défectueux » de la périphérie, l'autoconstruction cohabite avec l'agencement géométrique de l'espace: il suffit d'observer une vue aérienne de n'importe quel quartier de la Zone Ouest (Figure 1), souvent considérée comme " la périphérie de Rio de Janeiro ", pour avoir une idée nette de longues rues rectilignes, qui se croisent en angle droit ; de ces pâtés de maison avec leurs constructions bien rangées de chaque côté ; de ces îlots (quadras) disposés à intervalles réguliers, de tailles souvent comparables sans être pour autant uniformes. Avec un regard attentif, on devinera que tout l'espace n'a pas été loti en une seule fois, qu'il y eut plusieurs opérations de lotissement, obéissant à des règles d'urbanisme différentes. Il y a comme une " apparence d'ordre » dans ces lieux, qui les distingue de n'importe quelle occupation dite " spontanée », comme il en va pour les favelas - l'Institut Brésilien de Géographie et Statistique (IBGE), l'équivalent brésilien de l'Institut National de la Statistique et des Études Économiques (INSEE), définit les " agglomérations sous-normales " (aglomerados subnormais), synonyme de favelas, comme des ensembles de maisons " disposées, en général, de façon dense et désordonnée ", qui ont été urbanisés " en dehors des standards en vigueur ", d'où " des voies de circulation étroites et d'alignement irrégulier, des parcelles de tailles et de formes inégales ${ }^{7}$.

\footnotetext{
5 Encore aujourd'hui, les habitants de ces lotissements non-urbanisés recourent fréquemment à l'entraide pour construire les équipements collectifs auxquels ils n'ont toujours pas accès. Dans le lotissement du Jardim Maravilha par exemple, le plus vaste loteamento irregular de Rio de Janeiro, les habitants eux-mêmes creusent sur les bas-côtés des fossés par où s'écoulent les eaux usées (les funestes valas negras, les " fossés noirs "), souvent à la sueur de leur front, parfois en louant les services d'une tractopelle. Il leur arrive aussi d'acheter ensemble les tuyaux nécessaires à la construction des canalisations d'eau ou les poteaux qui leur permettront de raccorder leur logement au réseau électrique. Les habitants appellent mutirão cette forme d'entraide qui implique une participation à la fois conjointe, égalitaire e non-rémunérée à une activité d’utilité collective. Sur les différentes modalités d'urbanisation dans le cas spécifique du Jardim Maravilha, je me permets de renvoyer à ma thèse de doctorat (Cortado 2018).

6 On a préféré l'expression de « lotissement défectueux " à celle, plus littérale, de « lotissement irrégulier " pour traduire loteamento irregular: nous souhaiterions suggérer ainsi un parallèle, sur lequel nous ne pourrons malheureusement nous attarder, entre la situation des lotissements périphériques de Rio aujourd'hui et celle des lotissements parisiens ou lyonnais dans les années vingt et trente, à l'époque de la " crise des lotissements " (Fourcault 20oo). " Lotissement défectueux » était alors l'expression consacrée dans la presse et la littérature urbanistique pour se référer à ces lotissements de " mal lotis », aux constructions éparses, envahis par la boue et la végétation.

7 D’après la définition officielle adoptée lors du dernier recensement (2010).
} 
Figure 1: Vue aérienne d'une partie de la région de Campo Grande, dans la Zone Ouest de Rio. La zone entourée d'orange correspond au lotissement Vila Califórnia (Figure 2).

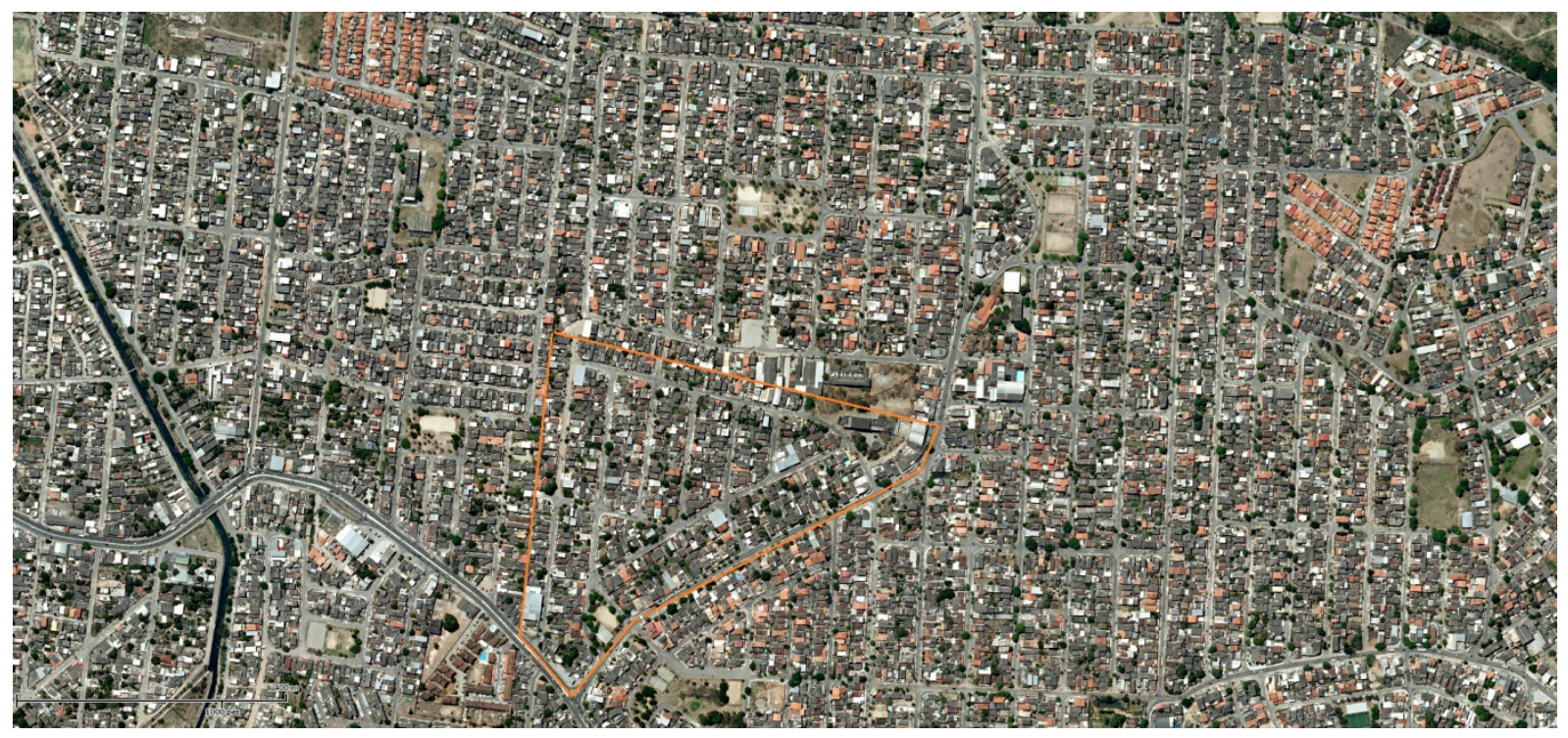

Sources: Instituto Pereira Passos (IPP), Prefeitura da Cidade do Rio de Janeiro (PCRJ).

Il y a une longue tradition de réflexion dans les sciences sociales sur l'agencement géométrique des espaces, l'adoption de plans orthogonaux aussi appelés grid par les anglophones (Rose-Redwoord: 2008). Des philosophes et sociologues comme Michel de Certeau et Henri Lefebvre ont proposé, dans les années soixante-dix, une théorie critique de ces agencements géométriques: pour le premier, ces agencements exprimaient une " pulsion scopique et gnostique ", la fantaisie de dominer le monde visuellement et intellectuellement (De Certeau 1990: 39-42); pour le second, l'espace produit par le capitalisme se caractérisait par la " primauté du visuel (du géometrico-vidéo-spatial) ", la géométrie étant utile aux planificateurs pour dominer visuellement l'espace, concevoir des " plans " justement et ainsi l'adapter aux besoins du mode de production dominant (Lefebvre 1999: 326-331). Tous les deux postulaient donc un lien étroit entre géométrie, visualisation et domination, politique et intellectuelle. Les analyses de Timothy Mitchell sur l'urbanisme colonial égyptien reposent plus ou moins sur ce même postulat puisque l'agencement géométrique de l'habitat est défini par cet auteur comme une " méthode d'encadrement " (enframing), destinée à confiner, discipliner et surveiller, sur la base d'une " apparence d'ordre " (appearence of order): en géométrisant l'espace, on impose un ordre aux apparences qui est tout autant une apparence d'ordre (Mitchell 1991: 6o). De même, pour James Scott, le plan orthogonal faisait partie de ces instruments utilisés pour rendre la ville « lisible », pour « disposer la population de façon à simplifier les fonctions classiques de l'État comme la levée des impôts, la conscription et la prévention des rébellions » (Scott 1998: 2).

Ces interprétations critiques des agencements géométriques ont le mérite de renvoyer l'aménagement de l'espace urbain aux « déterminations politiques englobantes ». Cependant, on peut encore se demander s'il ne conviendrait pas de considérer la critique de Santos dans le cadre de ce travail: ne serions-nous pas en train de réduire la forme de l'espace à des causes « un tantinet abstraites », en voulant expliquer l'agencement géométrique de l'espace par un besoin de l'État de rendre la société « lisible » ? Comment fait-on concrètement pour " lire » une société et, au-delà de la métaphore, on peut se demander qui lit quoi ? Il faut ici rendre justice aux documents d'urbanisme, en particulier aux cartes, schémas et plans, car c'est par ces « artefacts graphiques » (Hull 2012) que les agents publics et privés visualisent et organisent 
quotidiennement « la production de l'espace » urbain. Par-delà les « artefacts » les plus grandiloquents tels que les "schémas directeurs ", qui monopolisent souvent l'attention des spécialistes, il y a tout un continent de « pratiques documentaires prosaïques au travers desquelles la ville est construite, régulée et habitée " (idem: 4). Alors qu'il enquêtait sur l'administration urbaine d'Islamabad, Hull se rendit compte de ce que « l'aménagement et la régulation de l'espace étaient effectués au travers de millions de documents plus modestes, tels que les dossiers, les plans de maisons et les formulaires " (idem: 58). Il a alors critiqué le mythe du panoptique ou de «l'Cil de Dieu » qui imprègne la réflexion sur les rapports entre l'État et l'espace: « toutes les techniques administratives ne convergent pas vers un ensemble unifié de représentations. Elles génèrent des agrégations d'artefacts aux perspectives variées et limitées qui souvent sont difficilement commensurables " (idem: 181). Cette " formulation perspectiviste " (perspectivist formulation) ne signifie en rien une dissolution des questions politiques dans des questions techniques, mais au contraire une extension de ces questions politiques aux questions techniques, sous la forme d'une " microphysique du pouvoir ", "d'une attention au complexe formé par les relais et interdépendances qui permettent aux programmes de gouvernements d'agir et d'intervenir sur les lieux, personnes et populations qui sont leur problème " (Miller \& Rose 1990: 8).

Il se trouve que les lotissements de Rio de Janeiro dérivent d'un " artefact graphique » (Hull 2012) appelé « Projet Approuvé de Lotissement » (PAL): il s’agit essentiellement de plans (Figure 2) sur lesquels figurent, numérotés et mesurés, rues, îlots et parcelles, de même que les signatures du propriétaire et de l'autorité municipale responsable pour l'approbation du projet. Avant d'exister physiquement dans l'espace urbain, les lotissements possèdent donc toute une existence documentaire dans une temporalité durant laquelle se construisent les relations entre les acteurs impliqués dans les opérations de lotissement: d'après la législation encore en vigueur aujourd'hui, le propriétaire du terrain à lotir doit soumettre aux autorités municipales un projet de lotissement, que celles-ci approuvent ou non ; les parcelles sont ensuite démarquées conformément au plan qui a été approuvé, lequel sert aussi à identifier chaque propriété lors de l'enregistrement des transactions chez le notaire. En d'autres termes, le PAL s'impose comme une « technologie » indispensable pour coordonner les différents acteurs du lotissement. Sur la base de recherches que nous avons menées sur les documents et le code de l'urbanisme à Rio de Janeiro nous prétendons faire ici la " généalogie " (Foucault 2004) de cette technologie, en identifiant les rationalités techniques et politiques qui ont présidé à son invention. Il ne s'agit donc pas, à l'instar des interprétations diffusionistes du plan orthogonal (Rose-Redwood 2008), de découvrir l'origine historique du lotissement, ni même de révéler une quelconque formule idéologique à l'œuvre derrière cette pratique. Ce qui nous intéresse en premier lieu, c'est le rôle du lotissement dans la " problématisation " de l'espace urbain, le rôle du lotissement en tant qu'outil d'action sur l'action des individus engagés dans la production de l'espace urbain - la dite périphérie se révèle justement l'un des principaux enjeux de cette problématisation. Ainsi, notre contribution s'inscrit tout autant dans le cadre des governmentality studies que de l'ethnographie des documents: nous soutenons en effet que la « raison graphique " (Goody 1979) à l'œuvre dans les documents d'urbanisme a joué un rôle éminent dans la conception et la résolution des problèmes liés au gouvernement de l'espace urbain. Il se trouve en outre que les deux aspects essentiels du lotissement, la création de voirie et la division du sol en parcelles, n'ont pas toujours reçu la même attention des pouvoirs publics: si dès le début de la colonisation le gouvernement a subordonné la création de voirie (arruamento) à des normes précises, la première réglementation (municipale) des opérations de division foncière en milieu urbain date des années trente. On commencera donc par décrire ces techniques visant à contrôler d'abord la création de voirie, en insistant sur le rôle des plans, des registres et des permis. Ensuite, on abordera la législation moderne sur l'alignement (alinhamento), cette pratique administrative qui consiste à démarquer la frontière entre le domaine, public, de la rue et celui, privé, des propriétés, car c'est de cette législation qu'est né 
tout l'appareil juridico-administratif en charge aujourd'hui du lotissement. Enfin, on s'intéressera à la réglementation du lotissement proprement dit, telle qu'on la trouve dans le « Code Godoy » de 1931 et le Code de la Construction de 1937.

Figure 2: PAL 16.386. Projet du lotissement Vila Califórnia, dans le quartier de Campo Grande (Figure 1), approuvé en 1951.

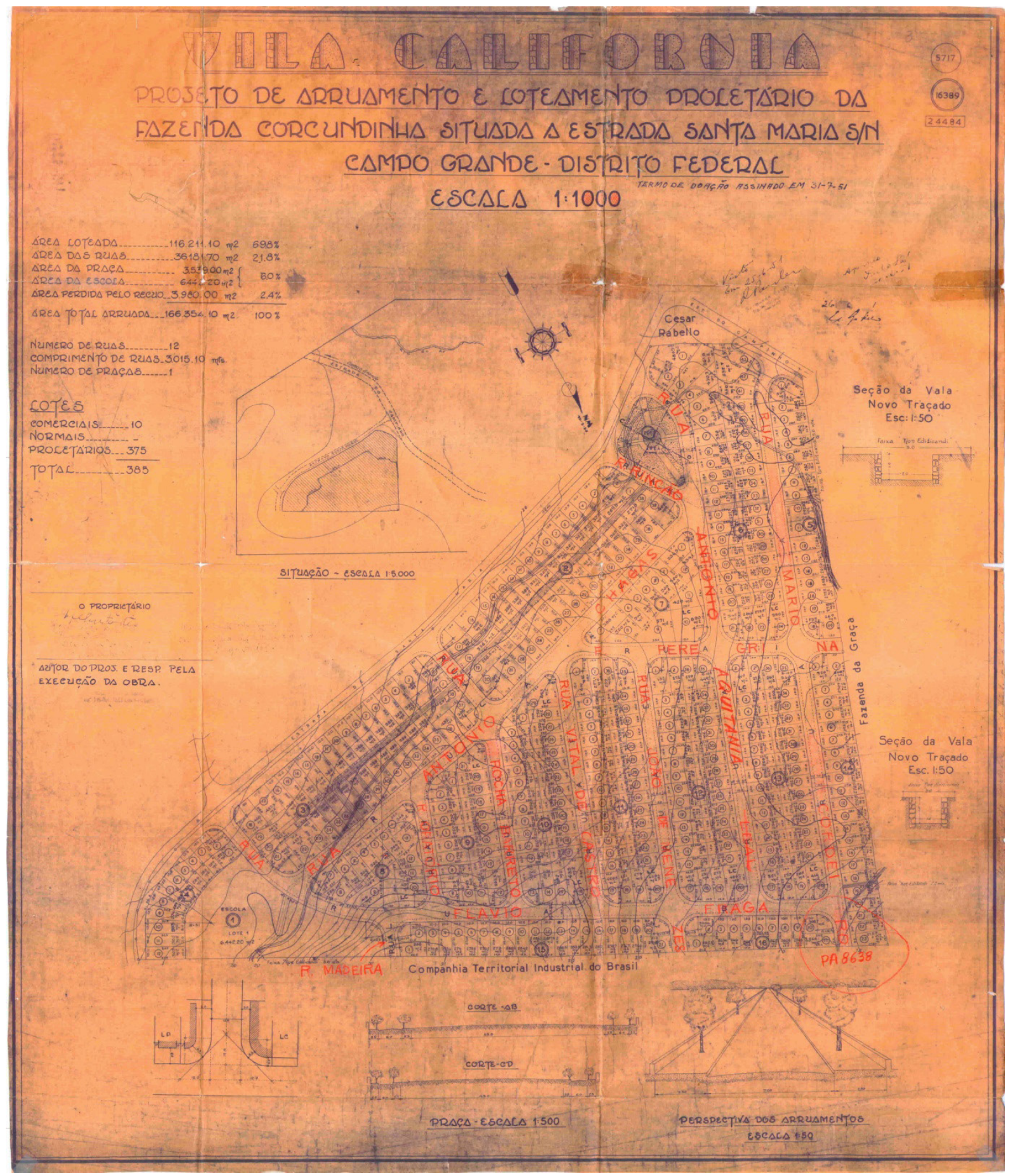

Source: Secretaria Municipal de Urbanismo (SMU), PCRJ. 


\section{Permission de voirie et defense des espaces publics}

Au temps de la colonisation, deux institutions spécifiques, l'almotaçaria e l'arruação (ou " acte de voirie »), exerçaient un certain contrôle sur les constructions en milieu urbain. Héritage de la colonisation islamique, l'almotacel était un fonctionnaire élu par la Chambre Municipale qui surveillait, entre autres attributions, les constructions des particuliers, ordonnant la démolition de celles qui encombraient les voies de passage et de celles qui contrevenaient au droit de servitude, lequel fixait les obligations des voisins les uns par rapport aux autres en matière d'édification. L'almotaçaria fonctionnait comme une institution médiatrice des conflits entre l'oikos et l'urbes, mais cette médiation ne survenait qu'a posteriori, après sollicitation de l'almotacel par les riverains eux-mêmes (Pereira 2001). Ce n'était déjà pas le cas des actes de voirie. À partir du quinzième siècle, l'administration portugaise se mit à réprimer vigoureusement l'usurpation des espaces publics, ces édifices qui non seulement barraient la circulation, mais s'accaparaient illégalement une certaine fraction de la voie publique. Obstacle au transit des marchandises, l'étroitesse des voies s'affichait comme l'autre mal à combattre - les premières grandes opérations d'élargissement de voies publiques au Portugal datent de la fin du quinzième siècle. Qui plus est, en accord avec les maximes esthétiques de l'époque, le pouvoir royal se montrait toujours plus préoccupé par l'apparence des rues ${ }^{8}$. En conséquence, le gouvernement prit plusieurs mesures pour contenir les avancées des constructions sur l'espace public et assurer ainsi la continuité des façades entre les édifices d'une même rue. Une en particulier joua un rôle fondateur dans l'urbanistique moderne: l'instauration par la Chambre de Lisbonne, en janvier 1504, d'une autorisation administrative préalable pour tous travaux de construction ou reconstruction d'immeuble. La Chambre déterminait ainsi que personne " n'ose abattre un mur, qu'il faille faire faire les fondations, sans l'avoir d'abord fait savoir à la chambre aux échevins pour qu'ils fassent voir et mesurer vers où iront les dites fondations ", et cela pour que, au moment de la reconstruction, " les murs ne puissent pas davantage occuper les rues et les passages ni ne leur prendre sinon ce qu'ils possédaient avant » (Pinto 2012: 160). Pour la première fois, le gouvernement conditionnait les opérations de construction en général à l'obtention d'une permission administrative, laquelle requérait inspection et mesure de la frontière entre la rue et l'immeuble. Ce procédé, baptisé de cordeamento ou " mise en corde " à Lisbonne puisqu'il nécessitait l'usage d'une corde, fut implanté à Rio dès les tout débuts de la colonisation, mais il y prit le nom de arruação ou arruamento, mot qui désigne l'acte (et le résultat de l'acte) de créer une voirie, mais aussi de distribuer physiquement des bâtiments des deux côtés de la rue.

L'ancienneté de cette pratique semble attestée par un acte de démarcation effectué le 4 janvier 1596, lequel indique que le propriétaire dénommé Fernão Baldes serait venu accompagné d’un greffier (escrivão) et du " voyer " (arruador) Francisco Gomes pour mesurer ses terrains et " laisser à la rue ce qui lui revient " (Anais da Biblioteca Nacional 1962: 251). Le voyer fixa une largeur minimale de 35 paumes (environ $7,7 \mathrm{~m}$ ) pour la nouvelle voie. Quelques années après, dans la Correction du 16 avril 1624, le juge João de Souza de Cardena " veilla à ce que personne ne fasse de maison en pierre et de torchis, sans que l'on fasse la voirie (arruar) par le Conseil, avec l'assistance d'au moins deux Échevins et d'un juge, ou à la place d'un Échevin, du Procureur, avec le Greffier du Conseil, et le Voyer pour qu'ils voient s'ils tordent ou enlèvent quelque chose à la Rue, sous peine de perdre tout son ouvrage et de démolition des maisons dans les termes dans lesquels elles se trouveront (...); il déclara encore que les rues se fassent droites, sans qu'elles ne prennent fin en aucun mur ayant la largeur de trente paumes " (Archivo do Districto Federal 1894: 399). Aussi, « faisait-on la voirie » (arruava) en des rues déjà ouvertes, pour empêcher que les reconstructions et les nouvelles constructions n'avançassent sur la voie ou ne la rendissent tordue, mais aussi en des zones vierges qui, appelées à accueillir de nouveaux immeubles, avaient besoin d'une voirie pour assurer la circulation

8 Jusqu'au dix-neuvième siècle, il n'était pas dans l'usage de reculer les immeubles résidentiels: on construisait directement sur l'alignement, de sorte que celui-ci se confonde avec la succession des façades 
future des résidents - ce qui fait sens vu que les limites de la rue coïncidaient avec celles des bâtiments. Dans les deux cas, la " permission de voirie " (auto de arruação) permit un contrôle a priori sur les travaux de construction. À partir du dix-huitième siècle, il incomba au greffier de consigner ces permissions de voirie dans des livres officiels. Il convient de rappeler qu'il n'existait encore aucune norme pour la largeur des parcelles à l'époque, d'où le fait qu'elle pouvait facilement varier de 3 à $13 \mathrm{~m}$ en fonction du nombre de bâtiments à construire (idem: 171-177).

La permission de voirie exprime, de la part de l'administration royale et coloniale, un intérêt précoce pour les problèmes de circulation: la rue ne se réduisait pas à une " voie bordée d'immeubles ", elle fonctionnait déjà comme " un canal aligné, autant verticalement, grâce aux façades droites sans corps saillants, qu'horizontalement, aucune maison n'ayant un avantage sur l'autre » (Pinto 2013: 161). La permission de voirie soumettait donc le contrôle de l'espace urbain à une nouvelle rationalité: il ne s'agissait plus seulement de réprimer des abus (obstruction de la voie commune, usurpation du domaine public), mais de les prévenir ; il ne s'agissait plus seulement de punir les infracteurs, mais d'orienter les travaux de construction en général, de sorte que les rues de la ville puissent former des canaux adaptés à la circulation des personnes et des marchandises. Loin d'être l'apanage du monde lusitain, ce passage de la répression à la prévention dans le contrôle des constructions survint simultanément dans d'autres pays européens, comme en France où la liberté de circuler et la commodité des habitants servirent aussi d'argument (Harouel 1977). On notera également que cette époque, les seizième et dix-septième siècle, coïncide historiquement avec le surgissement d'une toute nouvelle technologie politique en Occident: la " police ", laquelle visait à « manipuler, maintenir, distribuer et rétablir les rapports de force dans un espace qui implique des croissances compétitives » (Foucault 2004: 319).

\section{Artefacts graphiques et réforme urbaine}

De la nécessité de protéger le trafic, on en est venu aux dix-septième et dix-huitième siècles à celle de rectifier les rues, de faire en sorte que la ligne de démarcation entre la rue et l'espace privé fût droite. En plus d'améliorer la circulation, qui ne cessait de croître avec l'expansion des villes, une rue bien droite convenait au goût classique pour les formes géométriques et permettait l'uniformisation des façades: dans le langage de l'époque, la rectification des rues contribuait tout autant à « l'embellissement » des villes qu'à l'augmentation de "l'utilité publique " (Harouel 1977: 146). À Lisbonne, la municipalité se mit à exiger des propriétaires qu'ils avançassent leurs façades, pour donner à l'alignement une forme droite (Pinto 2016: 162). Si la rectification des rues s'avérait une tâche dispendieuse pour les coffres publics, car elle obligeait le pouvoir à indemniser les propriétaires à hauteur du terrain perdu, le tremblement de terre de 1755 engendra dans la capitale portugaise les conditions favorables pour une ample réforme du droit de voirie. D’abord, la reconstruction de Lisbonne fut placée sous la dépendance d'un Plan pour réguler l'alignement des rues et la réédification des maisons dans le centre-ville de Lisbonne, conçu en 1758 par l'équipe du " grandingénieur " (engenheiro-mor) Manuel da Maia, approuvé par le ministre Sebastião José de Carvalho e Melo, future Marquis de Pombal. Le plan venait accompagné d'un dessin représentant les travaux prévus par le plan et exécuté par les ingénieurs militaires Eugénio dos Santos e Carvalho et Carlos Mardel. Ce dessin révélait l'adoption d'une trame rigoureusement orthogonale, opposée aux ruelles étroites et sinueuses de l'ancienne ville. Le plan établissait également une hiérarchie entre les rues principales, qui atteignaient les 60 paumes de largeur $(13,20 \mathrm{~m})$, et les rues secondaires, qui faisaient 40 paumes $(8,8 \mathrm{~m})$. Il obligeait aussi les propriétaires affectés à terminer les travaux de reconstruction en cinq ans et « en conformité avec le Plan qui n’a pas seulement défini les alignements et les dimensions des parcelles, mais aussi la propre structure constructive des édifices, la hauteur sous plafond et le dessin des façades, en accord avec les projections 
exécutées par la Maison du Dessin (Casa do Risco) » (idem: 204). Avec ce plan de reconstruction, la construction dans le centre-ville de Lisbonne s'est retrouvée sujette, de façon systématique, à un ensemble de normes précises et, nouveauté, " d'artefacts graphiques ", car il n'était pas permis de construire différemment des plans officiellement approuvés et signés par le Marquis de Pombal lui-même.

Cette réorganisation autoritaire et généralisée de la construction ne fut possible que dans « l'état d'exception " permis par la catastrophe naturelle. Toutefois, avec la venue de la Cour Portugaise au Brésil en 1808 et l'élévation de Rio de Janeiro au rang de nouvelle capitale de l'Empire Portugais, les principes issus de l'urbanisme lisboète firent leur apparition sur le nouveau continent, ce qui se traduisit d'abord par la création d'une Intendance Générale de la Police en avril 1808, laquelle avait pour mission de civiliser et urbaniser la capitale - en plus de surveiller les mœurs, l'Intendance assurait l'entretien et l'amélioration des rues et réglementait les constructions, prohibant par exemple l'installation des jalousies, aussi appelées moucharabieh en raison de leur origine arabe (Gagliardo 2014). Cette nouvelle approche " policière " de la ville vint à la rencontre des inquiétudes croissantes de l'élite urbaine concernant la salubrité des environnements urbains pour mieux transformer la façon dont le pouvoir problématisait la création de voirie. En effet, depuis le début du dix-huitième siècle, une nouvelle représentation de la rue était en train d'émerger, celle d'un canal qui permettait la circulation non seulement des personnes et des marchandises, mais aussi de fluides tels que l'air et l'eau, essentiels à la propagation des maladies (Corbin 1982). La topographie irrégulière de la ville, en " fixant l'air ", concourrait à la diffusion des " gaz méphitiques ", altérant donc la santé des habitants. En 1820, dans ses Prolégomènes aux maladies chirurgicales du pays, le chirurgien de la Chambre impériale Domingos Peixoto disait des rues de Rio de Janeiro que, « d'un extrême à l'autre, elles montrent dans leur continuité de nombreux points d'élévation et de dépression, à quoi contribue le fait de ne point être correctement chaussées: par conséquent, toute la ville est marécageuse, les eaux des pluies qu'elle reçoit, ne parvenant pas à fuir vers la mer, faute d'écoulement, et d'aqueducs suffisants, s'éternisent donc et sont évaporées par la chaleur du soleil. [...] Les rues, en général, sont étroites, et cette condition fait que le mouvement des vents est peu favorable et que l'on sent, au maximum, la chaleur du sol » (Peixoto 2008: 99). Autrement dit, les vieilles rues du centre, étroites, irrégulières et sinueuses, présentaient le double défaut de faciliter la stagnation des eaux et d'empêcher la rénovation de l'air par le vent, favorisant ainsi la propagation des miasmes. De telles opinions issues des milieux médicaux autorisés avaient une profonde influence sur la perception de la ville par les élites à un moment où le pouvoir des médecins au sein de l'administration publique ne faisait que croître (Machado 1978).

Il n'y a donc rien de surprenant à ce que le Code des Postures (Código de Posturas) municipales de 1838 , la première compilation de lois municipales de Rio de Janeiro, contienne toute une série de dispositions relatives à la voirie. Dès le premier paragraphe de la seconde section' ${ }^{9}$, dédiée à l'exercice de la " police " (la première traitait de la santé), le Code prévoyait que « la Chambre [Municipale] dresse les plans suivant lesquels seront formés les rues, places et édifices dans la ville et son terme. Ces plans seront disponibles à la maison de la Chambre, et l'on en donnera des copies exactes aux contrôleurs et voyers des districts au sein desquels l'on ne peut édifier sans autorisation, permission de voirie et alignement ». Le paragraphe suivant stipulait que « au voyer il convient d'aligner et de profiler l'édifice, de réguler sa devanture, conformément au plan adopté par la Chambre ", vu que " personne ne pourra faire des travaux sur la devanture des immeubles sans autorisation de la Chambre " - pour les standards architecturaux de l'époque, la démarcation de la frontière entre les espaces publics et privés (alignement) se confondait avec la régulation des façades (profilage). En outre, aux nouvelles rues à délimiter s'imposait une largeur minimale de 60 paumes $(13,20 \mathrm{~m})$, identique aux « rues principales » de la Lisbonne pombaline. Enfin, le cinquième

9 Toutes les citations sont issues de PDF 1894. 
paragraphe obligeait les propriétaires, lorsqu'ils refaisaient la devanture de leurs immeubles, à les reculer ou bien à les avancer pour qu'ils " rentrent dans l'alignement ", suivant qu'ils excédaient le nouvel alignement ou se trouvaient en retrait par rapport à lui. Au travers de ces arrêtés, Rio de Janeiro se dotait donc d'une technologie administrative basée sur la production d'artefacts graphiques pour mieux contrôler la construction.

Quoique l'administration municipale n'ait gardé aucune trace de ces plans, qui n'ont probablement jamais vu le jour, au vu des tentatives ultérieures pour imposer de nouveau ces plans d'alignement, la tendance à la multiplication des artefacts graphiques ne cessa de se confirmer tout au long du dix-neuvième siècle. En 1856, la Chambre interdit toute édification ou réédification " sans que ne soit d'abord produit et soumis à l'approbation de la Chambre Municipale le dessin de la façade de l'édifice " (article premier de l'arrêté du 6 mai 1856), incluant ainsi dans le système des autorisations préalables la présence d'un nouvel artefact graphique, le dessin de la façade. À l'inverse de ce qui prévalait à Lisbonne au temps de Pombal, ce n'est pas l'administration qui fournissait au constructeur le dessin de la façade mais le constructeur lui-même qui devait lui soumettre le plan. À un contrôle de nature prescriptif s'opposait un autre de nature " proscriptif »: il ne s'agissait plus de contraindre le propriétaire à construire de la façon dont le voulait l'administration, mais plutôt de l'empêcher de construire de la façon dont elle ne voulait pas. Les instructions approuvées à la session de la Chambre du 11 novembre 1869 imposèrent ensuite la signature du propriétaire et du constructeur sur le dessin ; au permis de construire s'ajoutaient le terme de voirie et l'approbation, motivée par écrit, de ce dessin par l'ingénieur de district. En obligeant propriétaire et constructeur à signer les plans des façades, ces instructions mettaient en évidence le rôle éminent des artefacts graphiques dans le contrôle des différentes « agences » (agencies) engagées dans la construction. Progressivement, le contrôle des constructions se distinguait de celui exercé sur la voirie, ce que vint confirmer l'arrêté du 5 mai 1886, qui étendit l'autorisation préalable à « n’importe quelle construction à l'intérieur des terrains, même si le terrain présente une édification quelconque sur le devant »et à " n'importe quelles réformes ou travaux à l'intérieur des immeubles, à partir du moment où ces travaux atteignent plus de la moitié de l'aire occupée par l'immeuble ». Cette différentiation fait parfaitement sens au vu des deux grands changements de l'époque en matière d'architecture: en premier lieu, l'introduction progressive du recul, de façon à laisser un espace, souvent utilisé comme " jardin ", entre la rue et la maison (Reis Filho 2000: 48) ; en second lieu, l'extension du contrôle médical à l'intimité des maisons, d'où la condamnation, par exemple, des alcôves (Costa 1979).

L'arrêté du 15 septembre 1892, que l'on peut légitimement considéré comme le premier Code de la Construction de Rio de Janeiro, fixa définitivement cette dissociation entre voirie et édification. Les premiers articles de la loi énonçaient ainsi un certain nombre de règles pour encadrer les permis de construire, multipliant les contraintes en matière d'artefacts graphiques: la concession du permis requérait cette fois un dessin du terrain, un plan complet des travaux, ce qui voulait dire un plan pour chaque étage et un autre pour les façades principales ; les sections longitudinales et transversales des nouveaux immeubles; la projection des éventuelles dépendances (article second). Le troisième article considérait exclusivement les qualités graphiques des dessins, qui devaient être délivrés en duplicata, respecter une certaine échelle, être cotés et montrer la signature du propriétaire et du constructeur. L'édifice tout entier, et pas seulement la façade, se retrouvait sujet à permission, ce qui exigeait donc un certain effort de planification des travaux de la part des propriétaires et des constructeurs. Plus encore, la loi établit une nouvelle technologie pour réguler la création de voirie. D'après l'article sept, « il ne sera pas permis la division de terrains en places et rues, sans que ne soient préalablement présentés les dessins et profils longitudinal et transversal, cotés, à la municipalité, requise la permission nécessaire, laquelle, quoique gratuite, sera récusée si les rues et 
places présentent des angles dans leurs alignements ou ne sont pas dans les conditions de cet article et du précédent, ou s'opposent au plan général de la ville, organisée par la municipalité. La division des terrains cherchera toujours à s'approcher de la forme de rectangles peu allongés ".

Cet arrêté, promulgué par le Président du Conseil Municipal, le docteur Cândido Barata Ribeiro, contenait d'importantes innovations. En premier lieu, l'article sept sanctionnait la transition d'une modalité prescriptive vers une modalité " proscriptive » de contrôle: alors que dans les arrêtés du début du siècle, les nouvelles voies publiques devaient se conformer aux plans adoptés par la municipalité, dans le règlement de Barata Ribeiro il incombait aux propriétaires de soumettre leurs propres plans de voirie à la mairie, qui les approuvait ou non. Parmi les règles qui conditionnaient l'approbation du plan, il fallait que celui-ci soit en accord avec « le plan général de la ville, organisé par la municipalité », ce qui établissait donc une connexion entre deux artefacts graphiques, quoique le second fût encore en préparation. En second lieu, l'arrêté de 1892 exigeait la production de nouveaux dessins (profils longitudinal et transversal) de façon à contrôler non seulement la largueur (fixée directement à 13,20 m, sans référence aux unités de mesure coloniales) et la forme des rues (droites, de préférence), mais aussi leur régularité, sachant que les hygiénistes accusaient les variations dans le nivellement des rues de favoriser la stagnation des eaux (d'après le quatrième paragraphe de l'article six, il revenait au propriétaire de niveler la voirie et d'installer un caniveau de chaque côté des rues). Enfin, l'arrêté réglementait, pour la première fois, les divisions foncières, vu que les parcelles devaient posséder une forme rectangulaire peu allongée. Qui plus est, le paragraphe premier de l'article sept stipulait « qu'aucun lot de terrain avec une largeur inférieure à sept mètres sur le devant ne pourra recevoir une quelconque construction ". Cette intromission de la législation dans les opérations de division foncière s'étendit davantage encore avec le Décret n. 43 du 2 août 1893, dédié spécifiquement "à la création de voirie et au prolongement de celles déjà existantes »: en plus d'arrondir à $14 \mathrm{~m}$ la largeur des rues, le décret prévoyait qu'un vingtième des terrains supérieurs à $30.000 \mathrm{~m}$ revînt à la mairie pour l'aménagement d'une place. Le décret préconisait également que les rues, toujours droites, se croisassent en angle droit, situées à égale distance les unes des autres, et jamais à moins de $100 \mathrm{~m}$, ce qui revenait à imposer un plan rigoureusement orthogonal à la ville, du moins dans les zones encore dépourvues de voies publiques, et une taille minimale aux pâtés de maisons, qui ne pouvaient couvrir une aire inférieure à $10.000 \mathrm{~m}$.

Ces changements dans le contrôle de la division foncière reflètent ceux qui eurent lieu sur le marché immobilier de Rio de Janeiro à la même époque. Il faut se rappeler que, « jusqu’au milieu des années 188o, le processus de division du sol dans les banlieues (subúrbios) était principalement commandé par le petit propriétaire terrien, lequel divisait sa "ferme " (chácara) en parcelles, les vendant ensuite aux enchères " (Abreu 2013: 226). Dès la fin du dix-huitième siècle, des propriétaires fractionnaient leurs fermes en périphérie de la ville, dans le quartier de Botafogo par exemple, pour répondre à la demande des familles aristocrates, qui souhaitaient fuir un centre-ville surpeuplé. Cette expansion vers la périphérie reçut un coup d'accélérateur dans le dernier tiers du dix-neuvième siècle avec l'inauguration des premières voies ferrées (1858) et des premières lignes de tramway à traction animale (1868). Surtout, de nouveaux acteurs participèrent aux opérations de division foncière, tels que les banques et les compagnies immobilières, nationales ou étrangères, des acteurs détenteurs de capitaux en quête d'investissements lucratifs: avec eux, ces opérations se déroulèrent « de façon tout à fait nouvelle: ils n'ouvraient plus une ou deux rues ; ils créaient des quartiers entiers qu'ils vendaient en parcelles à terme " (idem: 26). Le cas le plus emblématique fut le surgissement du quartier Vila Isabel au début des années 1870: après avoir acquis de la famille royale les terres de la Ferme des Macaques, le Baron de Drummont fonda une société, la Compagnie Architectonique de Vila Isabel (1872), pour urbaniser la région ; il créa en même temps la Compagnie de Voies Ferrée Vila Isabel, pour relier le quartier au reste de la ville (Andreatta 2006: 144). De cette façon, 
les nouveaux acteurs du marché immobilier conféraient une valeur supplémentaire à la terre: « cela rend l'entreprise parfaitement viable, car la Compagnie Architectonique peut acheter les terres à bas prix, du fait d'un usage agricole stagnant, et les revendre à un autre bien supérieur, car il incorpore une nouvelle valeur d'usage crée: l'accès au centre de la ville " (Ribeiro 1997: 221). Ces nouveaux " lotissements » - le mot n'était pas encore entré dans l'usage, mais on parlait déjà de "vente en lots " (venda em lotes) ou de " lots de terre " (lotes de terreno) pour se référer aux parcelles - s'adressaient à une clientèle de classe moyenne, dont le pouvoir d'achat ne se comparait en rien à celui des aristocrates qui emménagèrent dans le sud de Rio de Janeiro au début du siècle.

Il y avait du côté des élites cariocas intéressées par les problèmes urbains une vraie préoccupation quant à l'essor de la ville. Cela apparut notamment dans les rapports de la Commission d'Améliorations (Comissão de Melhoramentos) installée par le Ministre de l'Empire João Alfredo Correia de Oliveira le 27 mai 1874, à laquelle participa l'ingénieur et futur maire de Rio Francisco Pereira Passos. On pouvait ainsi lire dans le second rapport de cette commission, chargée d'élaborer un plan pour l'aménagement de la capitale, que la ville de Rio de Janeiro « s'est étendue à mesure qu'augmentait sa population, prolongeant les anciennes rues jusqu'où elles pouvaient aller et en en formant de nouvelles sans aucune subordination à un plan préalablement étudié. Dans la majorité des cas, les alignements ont suivi la sinuosité des vallées, accompagnant le bas des collines. Dans d'autres, et ceux-là sont les plus récents, la seule loi qui préside à la direction des alignements est celle du caprice et de la convenance des propriétaires des terrains où l'on crée de la voirie. Nous ne citerons pas d'exemples: ils sont là disséminés parmi les divers quartiers de la ville, où presque toutes les semaines, on vend aux enchères des terrains à construire dans de nouvelles rues alignées et conçues par n'importe quel arpenteur (agrimensor), sans aucune sujétion aux principes qui devraient s'imposer dans ces cas " (CMCRJ 1876: 7 [souligné par l'auteur de cet article]). Les rapporteurs regrettaient que « dans ces nouveaux quartiers, la seule règle observée soit celle de donner aux rue la largeur minimale de 6o paumes marquée par la chambre municipale, sans avoir de respect pour tant d'autres conditions " (idem: 13). Il y avait donc un " problème du lotissement ", exigeant déjà l'intervention de la puissance publique, bien avant que la catégorie n'existe.

Pour les auteurs du rapport, la solution aux problèmes de voirie passait par l'adoption d'un " plan général ", d'un instrument administratif qui régulât la construction et la création de voirie à partir d'une vue d'ensemble de la ville et de ses perspectives de croissance. C'est que les problèmes de circulation, d'hygiène et d'infrastructure ne pouvaient se résoudre à l'échelle de chaque rue prise isolément vu qu'aucune n'existe indépendamment des autres: l'encombrement en un point donné de la ville affecte de proche en proche l'ensemble des voies. C'est pourquoi le " plan général " prétendait " discipliner les futurs actes de voirie, les nouvelles rues et avenues projetées [par le plan] servant d'artères principales, au milieu desquelles les propriétaires de terrains pourront créer d'autres rues secondaires, les soumettant à l'approbation de la municipalité » (idem: 33 [souligné par moi]). Autrement dit, le plan définissait les principaux axes, à l'image de cette vaste avenue de $40 \mathrm{~m}$ de largeur et de 4,8 $\mathrm{km}$ de longueur conçue pour relier le Campo de Santana en plein centre-ville de Rio à la jeune Vila Isabel, et laissait à l'initiative privée le soin créer la voirie secondaire en accord avec les besoins du marché immobilier - en connectant le centreville à sa frontière d'expansion, l'avenue en question, qui ne vit jamais le jour, dénotait déjà une aperception systématique de l'espace urbain, tout du moins de sa trame viaire.

Les deux rapports vinrent accompagnés d’un plan, « en deux feuilles de dessin à l'échelle 1:10o, distinguant à l'encre noire les constructions existantes et à l'encre carmin celles que nous projetons " (Figure 3), que la commission elle-même s'était donné pour toute première tâche de confectionner (CMCRJ 1875: 4). En plusieurs endroits, le second rapport demande au lecteur de " jeter un coup d'œil au plan de la ville » pour avoir ainsi une idée du « complexe de toutes les améliorations ». Parfois, le mot même de " plan ", comme souvent, renvoie tout autant à la liste des travaux prévus qu’à la carte utilisée 
pour les situer. C'est que les " plans » sont plus que des " projections »: du fait de leur « consistance optique " (Latour \& Callon 2015: 25), de leur façon d'organiser visuellement l'information disponible, ils possèdent une efficacité spécifique. En particulier, un plan permet d'embrasser immédiatement une vaste superficie de l'espace physique et d'apercevoir les rapports qui le composent - c'est sur le plan que le " complexe des travaux " se manifeste en tant que " complexe " du résultat attendu des opérations d'ouverture, d'élargissement, de prolongement ou de rectification des voies publiques, en même temps que l'on dispose d'un instrument pour mieux les contrôler. Le contraste entre le tracé du résultat projeté et celui de la trame existante, augmenté par le choix de couleurs opposées, indique les régions directement affectées par les travaux. Fondamentalement, le plan fonctionne comme le support d'une rationalité qui se représente l'espace comme un milieu d'interactions - on comprend mieux l'éminente participation des ingénieurs, entraînés à réaliser des croquis pour agir sur l'espace, dans l'institutionnalisation de l'aménagement urbain (Azevedo 2013).

Figure 3: Fragment du plan de Rio annexé aux rapports de la Commission d’Améliorations. À droite, le centre-ville de Rio qui donne accès à la Baie de Guanabara, à gauche, le jeune lotissement de Vila Isabel: on remarquera la large avenue de couleur rouge, parfaitement rectiligne, projetée pour relier les deux régions.

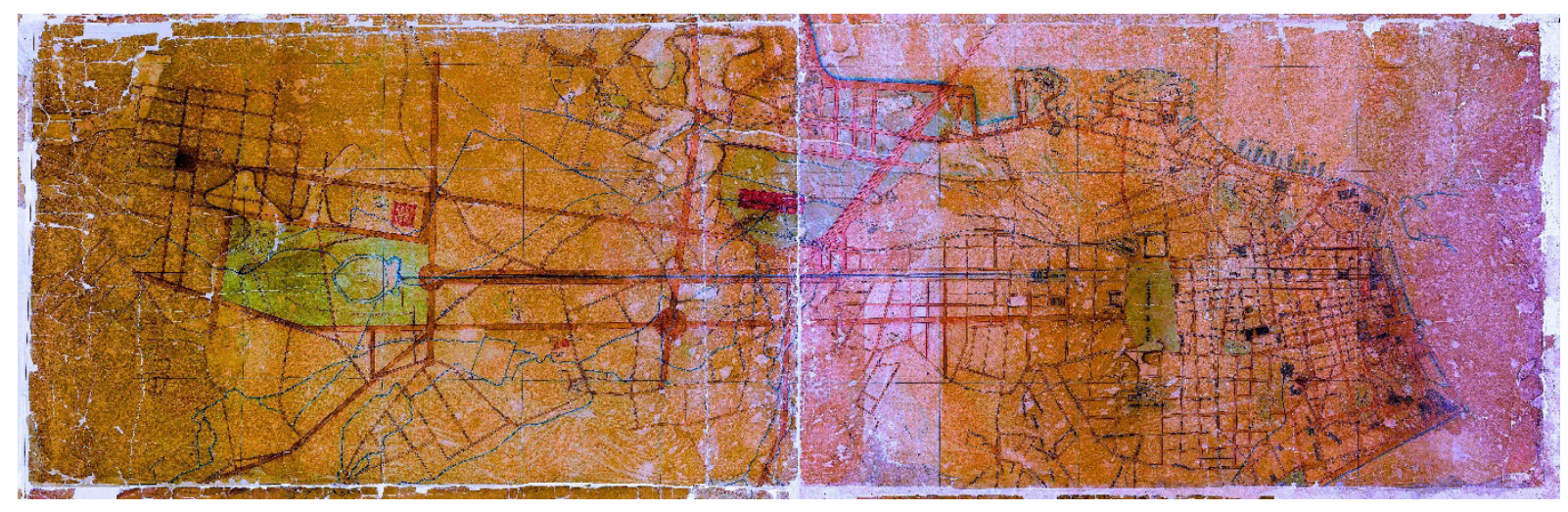

Source: Biblioteca Nacional (BN), 1875.

C'est donc sur la base d'artefacts graphiques que, trente années après, Pereira Passos ordonna la fameuse réforme urbaine (1902-1906) qui lui valut son surnom de « Haussmann tropical ». D’ailleurs, la réforme de l'infrastructure documentaire sur laquelle prenait appui l'administration municipale précéda la politique des grands travaux: alors qu'il n'était même pas encore maire de Rio, Pereira Passos réorganisa les services qui s'occupaient de ce que nous appellerions aujourd'hui l'urbanisme, nommant exclusivement des ingénieurs. Il mit au centre de sa nouvelle administration la Commission de la Carte Cadastrale, faisant d'elle une annexe de la Direction Générale des Travaux Publics et de la Voirie - sous la gestion de Barata Ribeiro (1892-1893) débuta le relevé de la carte cadastrale, à la suite du recrutement de Manoel Pereira Reis, professeur d'astronomie à l'École Polytechnique de Rio de Janeiro (Reis 1977: 8). La commission, sous la direction de l'ingénieur Américo de Sousa Rangel, avait d'ailleurs élaboré plusieurs " plans d'amélioration " (planos de melhoramentos) - le Décret du 20 décembre 1897 avait déterminé que, "pour toutes les concessions et contrats à célébrer avec la mairie, qui toucheront à la voirie, ne seront acceptés que les tracés projetés sur des plans qui procèdent de la Carte Cadastrale ", conférant déjà à la Commission un rôle important dans la gestion de l'urbanisation. Pereira Passos s'empressa d'utiliser les travaux de la Commission

10 Au sujet de l'écriture, Goody a montré comment elle met en évidence des rapports entre catégories qui difficilement apparaissent dans le discours oral, grâce à la « latéralité » du dispositif graphique (Goody 1979: 212). 
(idem: 17): l'importance que celle-ci a revêtue sous sa mandature nous rappelle que les plans ne font pas que projeter, ils suggèrent des interventions dans l'espace - les défauts d'une trame viaire ne sont jamais aussi apparents que sur un plan en vue aérienne. Enfin, Pereira Passos introduisit «l'uniformisation des projets d'alignement, aussi appelés projets d'améliorations, en feuille de toile impériale transparente, de 30 x $50 \mathrm{~cm}$. Les projets étaient visés par les ingénieurs, par l'Ingénieur Chef et Directeur des Travaux Publics, et ensuite approuvés par le maire » (idem: 18). À partir de cette normalisation des projets d'alignement commença la série de documents connue aujourd'hui sous le sigle de PAA ou « Projet Approuvé d'Alignement ». Le premier PAA représente ainsi l'Avenue Salvador de Sá dans le centre de Rio, œuvre de la mairie elle-même (Figure 4). En tout, la gestion Pereira Passos approuva près de 196 projets d'alignement, toujours en rapport avec des travaux commandités par la municipalité elle-même, telle que la construction des avenues Mem de Sá et Beira-Mar. Sous les administrations suivantes, les mêmes plans servirent à encadrer l'initiative privée: les opérations de lotissement, dans la mesure où elles créaient de la voirie, apparaissant alors en tant que " projets de voirie " (projeto de arruamento), lesquels faisaient partie de la série des PAA (Figure 5).

Figure 4: PAA 1. Projet d’ouverture de l’Avenue Salvador de Sá dans le centre de Rio de Janeiro, approuvé en 1913.

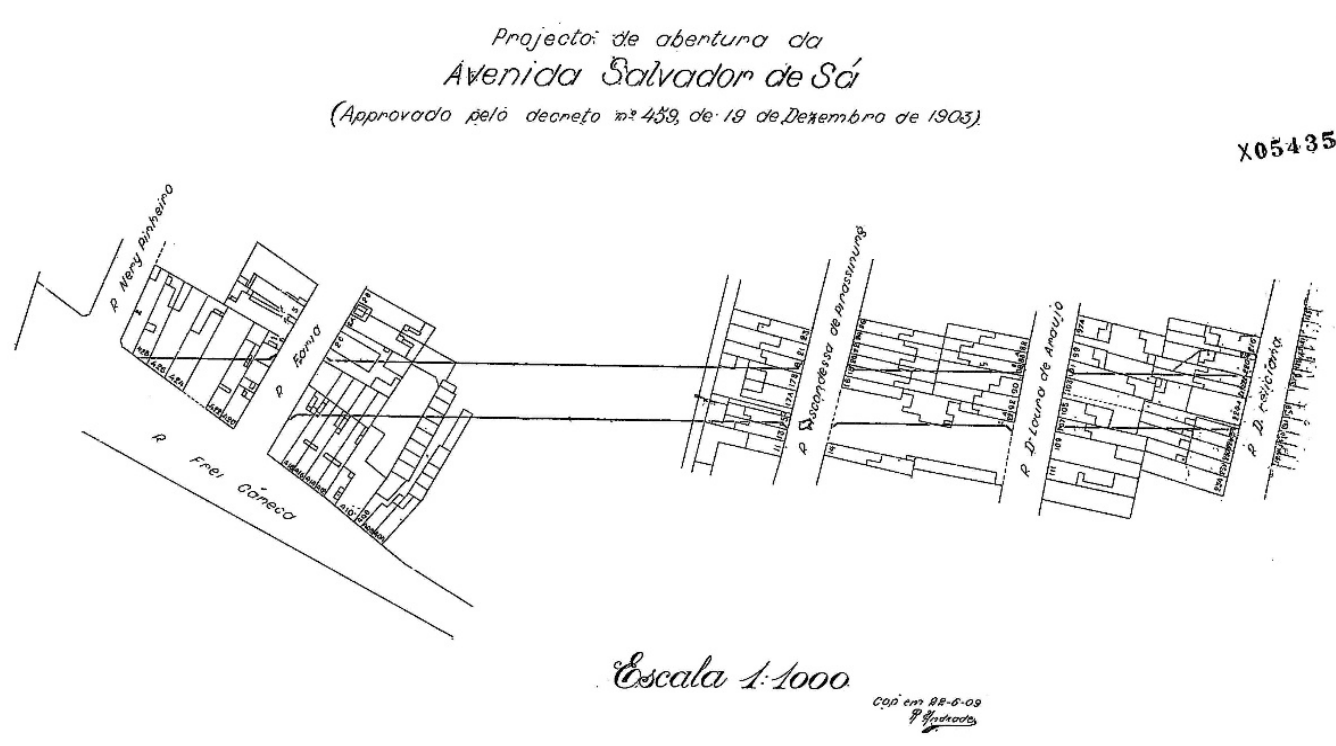

Source: SMU, PCRJ. 
Figure 5: Feuille n. 2 du PAA 1.426. Projet de lotissement dans la Zone Nord de Rio, approuvé en 1921.

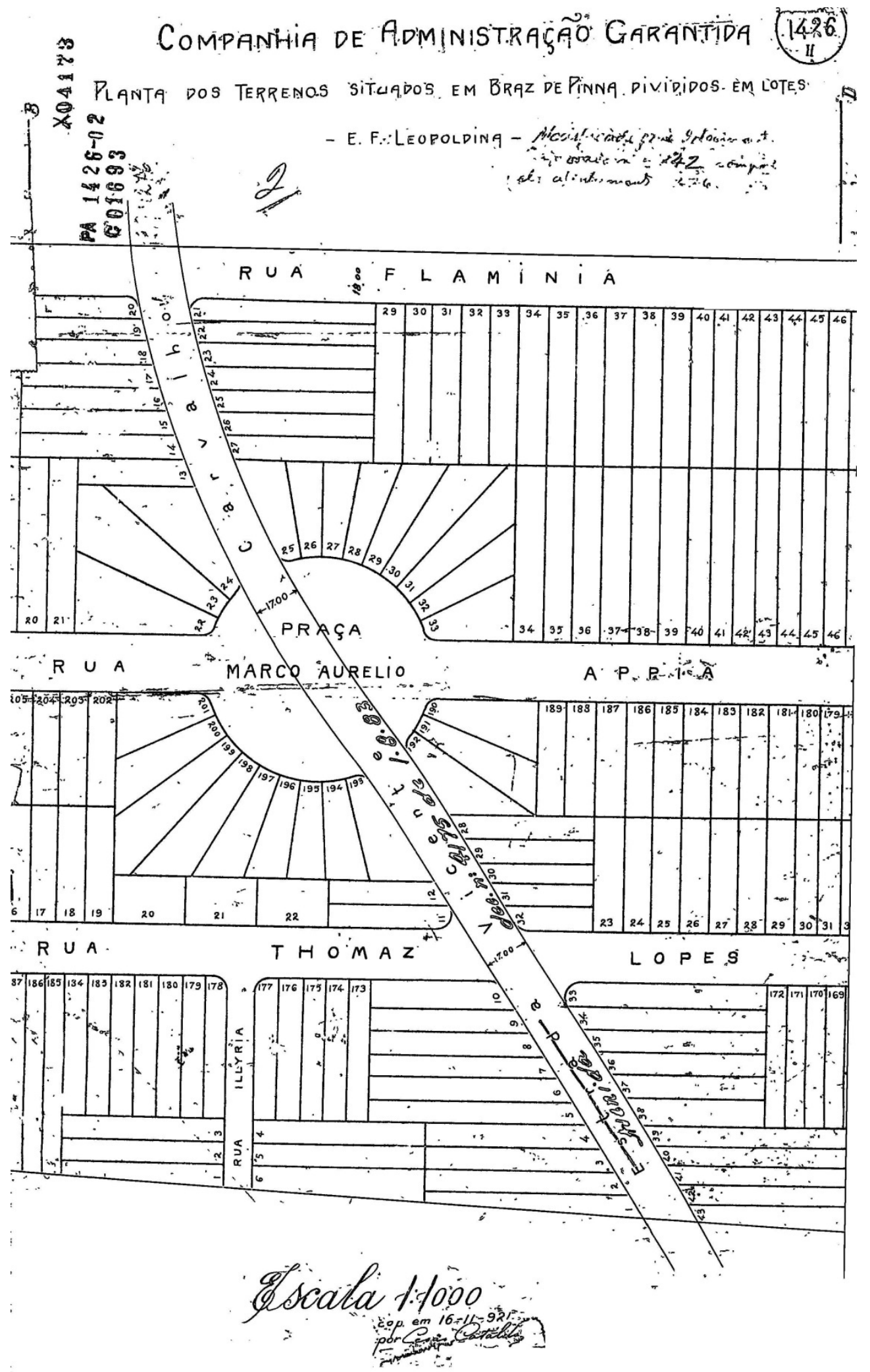

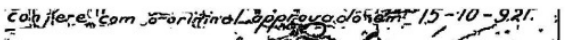

Source: SMU, PCRJ. 
La réforme de l'infrastructure documentaire permit l'organisation de toute une « économie politique du papier " (Hull, 2012) autour de l'alignement. Premièrement, avec l'uniformisation des PAA, la fixation des alignements, qui autrefois découlait du profilage des édifices, se mit à procéder d'un objet graphique: une figure géométrique (ligne rouge) sur un dessin (noir). Autrement dit, la rectification de la maille urbaine commençait par le tracé d'une ligne droite sur un dessin. Deuxièmement, les plans d'alignement dérivaient invariablement d'un autre artefact graphique: la carte cadastrale. Cet " allongement des réseaux sociotechniques " (Latour 1987) fit de la mairie un véritable " centre de calcul » urbanistique, un pôle pour agir à distance sur la trame viaire de la ville (idem: 222): comme la carte cadastrale offre une vue synoptique de l'espace urbain, l'alignement devenait fonction de cette vue synoptique, rendant ainsi possible une plus grande cohérence dans les opérations d'urbanisme, par rapport à des plans isolés. Troisièmement, la numérotation et l'archivage des PAA rendit possible l'établissement de " connections horizontales " (idem: 244) entre les PAA (un PAA peut faire référence à un autre PAA) ou entre les PAA et d'autres documents tels que les " termes de cession ", qui fixent les devoirs des lotisseurs en matière d'urbanisme et garantissent le transfert des rues et des places au domaine public. Quatrièmement, le jeu des visas (de la Commission de la Carte Cadastrale) et des signatures (du chef de la Direction Générale des Travaux Publics et de la Voirie et du maire) scella une coordination spécifique entre les bureaucraties municipales.

\section{Lotissement et planification des besoins collectifs}

Si, dès les débuts de la colonisation portugaise, le pouvoir s'est efforcé de contrôler la création de voirie, on ne peut pas en dire autant de la division du sol urbain - le mot même de " lotissement " n'apparaît dans aucun texte de loi avant le début des années trente (Sampaio \& Lira 2010: 674). Il existait bel et bien une réglementation des transactions immobilières: la Loi des Terres de 1850 imposa l'achat comme la principale modalité d'accès à la propriété foncière, l'enregistrement de chaque transaction à l'écrit, dans des livres paroissiaux au début, par des actes notariés ensuite. Cependant, la formation des parcelles urbaines ne dépendait d'aucune norme particulière, laissant aux agents économiques une liberté totale en la matière. D'où l'absence de régularité dans les découpages fonciers: les pâtés de maison prenaient souvent l'apparence de trapèzes dont les dimensions variaient de 40 à $60 \mathrm{~m}$ en longueur et de 50 à $100 \mathrm{~m}$ en hauteur ; les parcelles faisaient de 3 à $12 \mathrm{~m}$ de largeur pour 20 à $50 \mathrm{~m}$ de profondeur. La forme étroite et allongée de ces parcelles obéissait alors à une logique économique, l'occupation des alignements par les façades renchérissant les constructions à mesure qu'augmentait la largeur des parcelles. Et ce non seulement dans le centre de Rio mais aussi sur les nouveaux fronts de l'expansion urbaine. Le plan d'un petit lotissement situé dans le quartier d'Engenho de Dentro, inauguré au début des années 188o, fait apparaître des parcelles de moins de $11 \mathrm{~m}$ de largeur pour plus de 60 de profondeur (Figure 6). Le gouvernement fit alors très peu pour réglementer les divisions du sol urbain, quoique Barata Ribeiro introduisît la limite basse de $7 \mathrm{~m}$ de largeur pour pouvoir construire sur une parcelle. Ce fût seulement dans les années vingt et trente du siècle suivant que la puissance publique légiféra sur la division du sol urbain. 
Figure 6: Plan et catalogue des parcelles mises en vente par Silva Guimarães en 1880, dans le quartier d'Engenho de Dentro, Zone Nord de Rio de Janeiro.

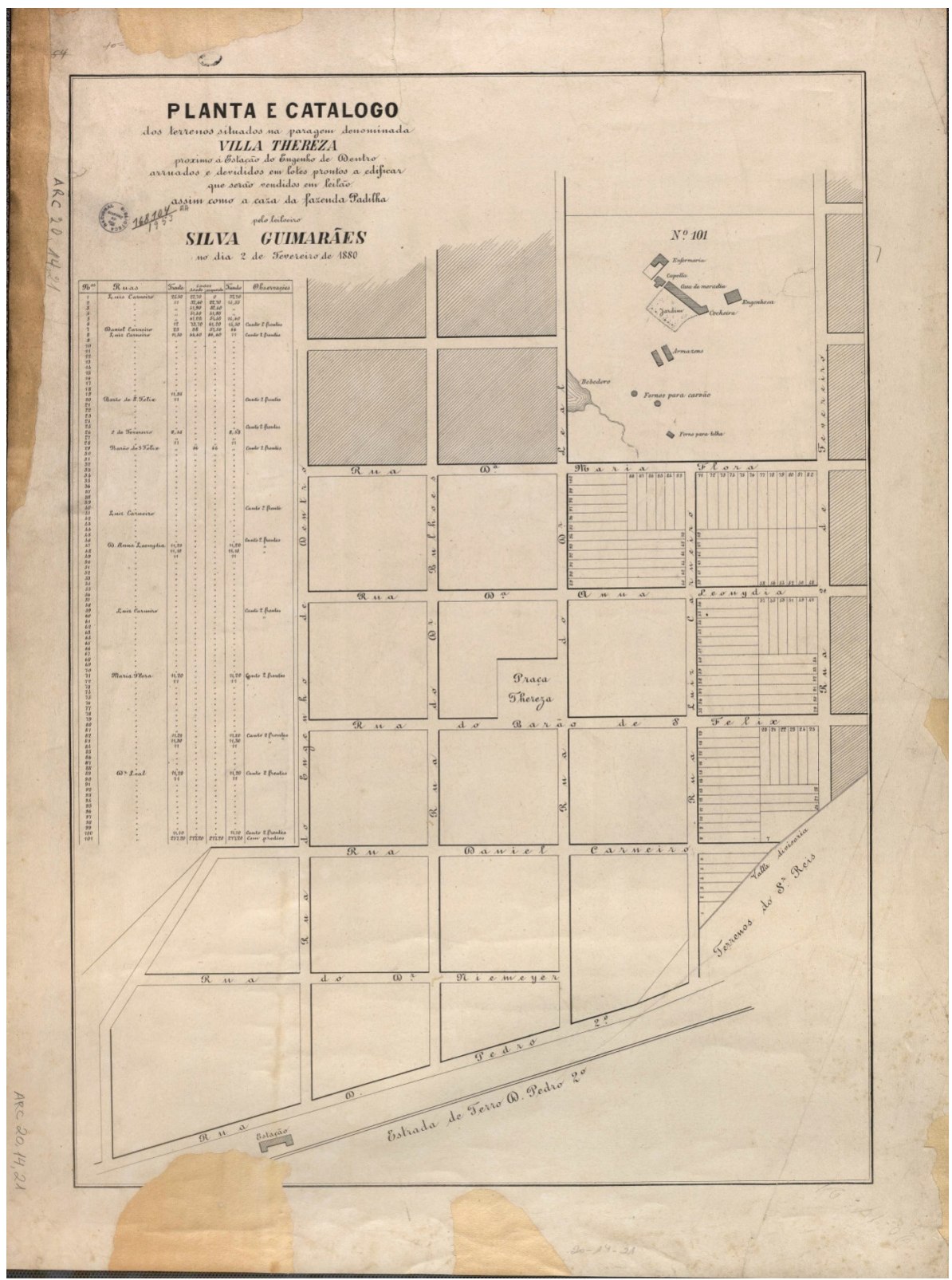

Source: BN.

La lenteur de la puissance publique a de quoi surprendre quand on la compare à l'intensité du phénomène. Entre 1870 et 1920, le nombre de voies publiques à Rio passa de 503 à 3.534 (Ribeiro 1997: 166, 178) ; entre 1890 et 1906, la croissance démographique des deux quartiers périphériques de Inhaúma et Irajá, où se concentraient les nouveaux lotissements, représentait plus de la moitié de celle enregistrée dans toute la ville (idem: 180). Rien que dans le quartier de Irajá, la population augmenta de $263 \%$ entre 1906 et 1920 , sans parler du nombre de voies publiques, qui passa de 81 à 741 (idem: 178; Abreu 2013: 82), indiquant ainsi une occupation préférentiellement horizontale de ces nouveaux espaces. La conception bourgeoise, qui difficilement tolère une quelconque intromission de la puissance publique dans les affaires de propriété, explique peut-être cette absence de contrôle sur la formation des parcelles, d'autant que les grands artisans de l'aménagement, les ingénieurs, appartenaient à cette couche sociale. Pour autant, une telle conception n’a pas empêché l'administration municipale d'exiger, à la toute fin du dix-neuvième siècle, le transfert 
vers la municipalité d'une partie des terres à lotir, en vue d'aménager une place publique. C'est qu'une place répondait à l'époque à des problèmes précis: la ventilation, afin de prévenir la propagation des maladies, et «l'embellissement " (embelezamento) de la ville, désormais soumise au goût des élites. Pour cette raison, moins que d'un crédo libéral dont l'histoire a prouvé l'impressionnante plasticité idéologique, l'absence de contrôle sur la division du sol nous semble plutôt signifier l'inexistence d'une problématique touchant spécifiquement au fractionnement des propriétés. Dans le cas de Rio de Janeiro, l'on doit à un éminent fonctionnaire du Département d'Ingénierie de la Direction des Travaux Publics et de la Voirie, par ailleurs pionnier de l'urbanisme au Brésil, cette problématisation de la division du sol: Armando Augusto Godoy.

Formé en ingénierie à l'École Polytechnique de Rio de Janeiro en 1904, Godoy connut une longue carrière au sein de la mairie de Rio, où il dirigea plusieurs commissions importantes pour l'urbanisme carioca, telle celle responsable de l'élaboration du Code de la Construction de 1924 (Silva 2003). C'est également lui qui invita l'architecte français Alfred Agache au début des années vingt pour concevoir le premier schéma directeur de la capitale. De façon générale, Godoy s'est efforcé de diffuser au Brésil les « idées nouvelles » de l'urbanisme: en 1927, il participa à la fondation de l'Association Brésilienne de l'Urbanisme ; il présida la première Commission du Plan de la Ville en 1931 ; il travailla comme consultant sur la construction de Goiânia entre 1933 et 1936. Godoy avait de l'urbanisme la vision d'une science " holiste ". En épitaphe à son recueil d'articles, L'Urbs et ses problèmes, Godoy définissait l'urbanisme comme « l'ordre et l'harmonie entre tous les éléments de la ville, les souterrains, les superficies et les élévations ; la bonne utilisation des terrains, la diminution des inégalités sociales, la solution rationnelle au problème du logement ; des parcs, des jardins, des squares, des temples, des écoles, des fabriques, des hôpitaux, des bibliothèques, des musées et des moyens de transport bien distribués et localisés " (Godoy 1943: 4). L'urbanisme selon Godoy se voulait donc une science, positive et normative, des interdépendances qui organisent l'espace matériel de la ville. Quelques années auparavant, Godoy avait proposé une définition un peu différente de cette discipline, « comme étant la création de l'esprit humain au moyen de laquelle on vise l'important résultat suivant: l'utilisation rationnelle, esthétique, hygiénique et économique des terrains de la ville " (Godoy 1935: 711-712). Pour cela, l'urbanisme exigeait « le concours et les lumières de l'ingénieur, de l'architecte, du sociologue, de l'économiste, du paysagiste, du sculpteur et de l'hygiéniste " (idem: 716). Godoy concevait ainsi l'urbanisme comme une extension de la rationalité planificatrice à la totalité de l'espace urbain: non seulement aux rues, infrastructures et constructions résidentielles, mais aussi aux rapports existants entre elles ; pour " assainir » et « embellir » la ville, mais encore pour l'adapter aux besoins économiques et sociaux de la population. L'urbanisme participait alors du grand mouvement réformateur qui s'empara de la société brésilienne dans les années vingt, et qui se caractérisait par la généralisation de la rationalité planificatrice aux diverses dimensions de la vie collective (éducation, travail, logement, etc.), que Micheal Conniff baptisa mouvement de " planification sociale " (Conniff 2006: 159).

Du point de vue holiste défendu par Godoy, la division du sol s'avère un sujet central. En premier lieu, l'expansion horizontale de la ville porte atteinte à l'économie et à la salubrité: « je pense que le plus grand mal dont souffre cette métropole se trouve dans l'expansion excessive, irrégulière, antiéconomique et antihygiénique dont elle fut la victime (...). Antiéconomique parce que les prolongements que Rio reçut n'obéirent pas au critère permettant de réaliser une occupation rationnelle des terrains, de façon à ne pas surcharger exagérément les coffres municipaux avec de multiples services publics. Antihygiénique pour avoir éludé le problème des égouts " (Godoy 1932: 21). Le problème hygiénique, en vérité, découlait du problème économique. L'occupation clairsemée de la ville contribuait à ce que « le revenu provenant aussi bien de l'impôt local et des taxes sur la chaussée que d'autres taxes ne suffise au maintien, en de bonnes conditions, des services publics les plus indispensables " (idem: 1935: 715): d'un côté, la multiplication des bâtiments de petite taille, consécutive à la baisse de la densité, diminuait la recette fiscale par mètre 
carré de la municipalité ; de l'autre, l'expansion horizontale augmentait le coût des services, tels que l'entretien des rues, et donc l'installation d'infrastructures comme le tout-à-l'égout - d'où l'observation suivante de Godoy selon laquelle « l'un des aspects les plus importants que l'urbaniste doit regarder en face est l'économique " (idem: 173). Il était donc nécessaire de freiner l'expansion horizontale de la ville. En second lieu, la forme des parcelles influait aussi sur l'économie et l'hygiène de la ville. « Elle [la parcelle] est, de fait, l'élément fondamental et indivisible dont tout le reste dépend. Si ses dimensions ne sont pas convenablement établies et mises en rapport, en ayant en vue le destin qui leur est réservé, les résultats ne s'accordent pas avec les fins voulues " (idem 1943: 99). Des parcelles " très étroites et profondes " dégradaient l'hygiène de la maison: « un des grands défauts des grandes villes, malheureusement de quelques nouvelles au Brésil, ce sont les parcelles étroites, où difficilement se résout sans grand sacrifice de terre le triple problème de l'ensoleillement, de la ventilation et de l'éclairage " (A Noite 1923: 3). Qui plus est, en obligeant les propriétaires à construire sur l'alignement, la forme des parcelles incommodait du point de vue esthétique, hygiénique et moral ; à l'inverse, « les immeubles convenablement éloignés de l'alignement, deviennent plus distincts, s'améliorent en général du point de vue architectural, acquièrent une autre grandeur et constituent, de fait, une résidence plus tranquille pour se trouver moins exposé aux bruits, à l'air vicié et l'agitation des rues " (idem 1943: 147). Quant aux irrégularités dans le tracé des parcelles, elles compliquaient l'installation des infrastructures urbaines, en raison des interdépendances entre parcelles dans l'accès aux équipements collectifs: « les maisons sont liées les unes aux autres par les rues, par les canalisations d'eau et d'égout, par le réseau d'éclairage et de téléphone, entretenant des influences réciproques " (idem 1935: 718). Par conséquent, « la mauvaise division des terrains complique l'installation des services d'eau, d'égout, d'éclairage dans les sous-sols en même temps qu'elle complique les travaux sur la chaussée et l'arborisation " (idem 1943: 60). C'est pourquoi « sans une bonne fragmentation des terrains, une bonne orientation des rues et une distribution convenable de tous les éléments urbains, il n'est pas possible de résoudre, en accord avec toutes les exigences, le problème du logement " (idem 1935: 718).

Il existait néanmoins une solution aux problèmes de la division du sol: la cité-jardin, laquelle, d'après Godoy, représentait « la plus parfaite et la plus complète création urbaniste de l'époque » (idem 1943: 137). Conçu par le socialiste anglais Howard Ebenezer, le modèle de la ville-jardin prétendait résoudre la question du logement en conjuguant les avantages de la ville et de la campagne. Ce modèle consistait en une planification rigoureuse, presque malthusienne, de communautés autonomes capables tout autant d'intégrer les différentes fonctions de la vie urbaine (résidentielle, commerciale, industrielle, etc.) que de permettre à leurs habitants de jouir de la nature, réduisant ainsi les effets délétères de l'industrie sur la santé et le moral de la population. Il se concrétisa pour la première fois en 1903, avec la fondation de la ville de Letchworth au Royaume-Uni, sous la direction des architectes Raymond Unwin et Barry Parker. Quelques années plus tard, les mêmes architectes projetèrent le Jardin Amérique (Jardim América) à São Paulo: en plus de bénéficier de toutes les infrastructures urbaines (électricité, eau, égout, gaz, etc.), ce lotissement se caractérisait par des parcelles bien plus vastes que la moyenne, de $900 \mathrm{~m}$ au minimum, par le recul des bâtiments par rapport à la rue et aux propriétés voisines et par la présence de jardins, de larges avenues et rues arborisées (Pereira 2001: 42). La cité-jardin était donc déjà une réalité dans le Brésil des années vingt ou, tout du moins, le garden suburb, vu que le Jardin Amérique perdit rapidement commerces et écoles pour devenir un quartier exclusivement résidentiel, contredisant ainsi l'inspiration originale d'Ebenezer. Godoy souhaitait étendre ce modèle à tous les lotissements, y compris à ceux destinés à une clientèle populaire, défendant que « dans les dites villes destinées aux familles prolétaires, la mortalité est plus petite que dans les autres centres de population, la criminalité et les grèves ayant presque disparu " (Godoy 1943: 152) - il faisait alors référence à la ville de Suresnes en France. Parmi les conséquences pratiques immédiates, 
il n'était plus possible de démarquer des parcelles qui se révélaient avoir moins de $6 \mathrm{~m}$ de largeur, car le modèle de la cité-jardin présupposait l'isolement de la maison au milieu de la parcelle, c'est-à-dire son éloignement des limites du terrain, suivant une ancienne revendication des hygiénistes.

Deux grands textes de loi, rédigés sous l'autorité de Godoy, signalèrent le début d'une réglementation portant sur la division foncière à Rio. Le premier fut le Décret n. 2.087 du 19 janvier 1925, le fameux Code de la Construction élaboré par la commission que dirigeait Godoy. Ce décret contenait d'importantes innovations, telles que la première définition juridique de la parcelle: « portion de terrain situé à côté d'une voie publique, décrite et assurée par un titre de propriété ». Toutefois, la plus grande innovation du décret en ce qui concerne la division du sol résidait dans l'article quatorze, car, en vertu du premier paragraphe, la municipalité ne délivrait de permis de construire qu’après « avoir soumis à l'approbation de la Mairie le plan de division de l'îlot respectif en parcelles ». De plus, le décret interdisait la division du sol "sans que le plan de cette division ne soit soumis à l'approbation de la Mairie " (second paragraphe); quant aux projets de voirie, ils devaient " contenir un plan de division en parcelles des terrains respectifs " (troisième paragraphe). Architectes et ingénieurs responsables de leur conception devait signer ces projets, du fait du paragraphe dix-sept. En d'autres termes, le décret instaurait, pour la division du sol, un permis semblable à celui qui existait déjà pour les constructions et reconstructions. Le second texte, le plus important dans la mesure où plusieurs dispositions du premier n'entrèrent jamais en vigueur, fut le Décret-Loi n. 3.549 du 15 juin 1931, aussi connu sous le nom de "Code Godoy »: il s'agit du tout premier texte de loi à prendre en compte tous les aspects du lotissement, depuis la création de voirie jusqu'à la division du sol. Ce décret-loi commençait en réaffirmant l'obligation de faire préalablement approuver les " projets " de " division des terrains en parcelles ou rues ", mais en ajoutant de nombreuses conditions à l'approbation, ayant trait à la confection d'artefacts graphiques. En premier lieu, il fallait désormais indiquer sur le dessin les connections avec les voies publiques, les canalisations d'eau et le réseau des égouts, la " situation schématique et les superficies maximales des futurs édifices sur les parcelles respectives » et les profils longitudinal et transversal de chaque rue (premier paragraphe) - encore une fois, on notera la ressemblance avec les procédés adoptés pour les permis de construire. En plus du dessin, le projet devait venir accompagné d'une déclaration détaillant l'ensemble des travaux à réaliser et d'un cahier des charges stipulant les servitudes pesant sur chaque parcelle. En second lieu, le décret imposait de nouvelles contraintes à la création de voirie: $26 \%$ de la superficie totale du lotissement devaient être réservés aux places et aux voies publiques (second paragraphe), la superficie moyenne des îlots allant de 10.000 à 12.00o m ～(ou bien, mais les deux règles s'équivalaient, les rues devaient être disposées « en moyenne, tous les $200 \mathrm{~m}$ dans un sens et tous les $60 \mathrm{~m}$ dans l'autre "); les rues résidentielles ne pouvaient posséder une largeur inférieure à $9 \mathrm{~m}$, alors que pour les voies dominantes (avenues) la limite s'élevait à $18 \mathrm{~m}$. En troisième lieu et pour la première fois, la loi fixait des dimensions minimales pour les parcelles: $9 \mathrm{~m}$ de largeur pour une superficie totale de $360 \mathrm{~m}$ (c'est-à-dire une profondeur moyenne de $30 \mathrm{~m}$ ). En quatrième lieu, « les terrains qui, de par leur situation, ne permettaient pas dans de bonnes conditions économiques " l'installation d'infrastructures urbaines, ne pouvaient " qu'être fragmentés en vastes parcelles, avec au minimum $50 \mathrm{~m}$ de largeur pour 100 $\mathrm{m}$ de profondeur ", de façon à dissuader le lotissement de propriétés difficiles à viabiliser. En dernier lieu, la vente et la location de terrains dépendaient de l'approbation des projets et de l'exécution des travaux de voirie et de canalisation d'eau et d'égout (cinquième paragraphe).

Ce règlement avait donc pour objectif d'obliger les lotisseurs à construire de véritables cités-jardins, des lotissements pleinement urbanisés, destinés à la construction de maisons isolées - la loi interdisait toute édification à moins de $3 \mathrm{~m}$ de l'alignement. Plusieurs dispositions dérivaient aussi du Plan Agache, dont Godoy fut l'ardent propagandiste: l'inclusion d'une déclaration de travaux (programa de obras) et d'un cahier des charges par exemple, ainsi que l'interdiction de mettre les parcelles en vente avant que le 
lotissement n'ait été approuvé par la mairie et complètement urbanisé (PDF 1930: LIV-LV) - clausule que l'auteur du Plan justifiait en référence à la loi française du 19 juillet 1924, conçue pour réprimer les abus de la part des lotisseurs peu scrupuleux. La règle des $26 \%$ (idem.: XVIII), la limite de 10.000 ou $12.000 \mathrm{~m}$ pour les pâtés de maison (idem: LII) et de 12 x $30 \mathrm{~m}$ pour les parcelles (idem: XIX) figuraient aussi dans le Plan: Agache expliquait que, parmi les $26 \%$ de superficie destinés à un usage public, $16 \%$ revenaient aux voies de circulation et 10\% aux places. Quant au minimum de 10 ou $12.000 \mathrm{~m}$, il provenait d'une étude réalisée par le Ministère de l'Hygiène anglais, qui adoptait un maximum de 30 immeubles par îlots (idem): sachant que la superficie moyenne des parcelles est de $360 \mathrm{~m}$, un tel maximum donnait un îlot de $10.800 \mathrm{~m}$, quelque chose entre 10.000 et $12.000 \mathrm{~m}$. Cette mathématisation ou géométrisation des relations spatiales, qui se retrouve aussi dans les normes de construction, interpelle: ne serait-elle pas le dernier prolongement de la " raison graphique », d'une représentation de l'espace fondée sur la médiation graphique (Goody 1979) ? Quoi qu'il en soit, plus que des mathématiques, ces relations mettent en évidence la problématisation conjointe de la parcelle, de la rue et de l'édifice, problématisation orientée par des considérations hygiéniques, esthétiques et économiques. Quant à la norme de 12 x $30 \mathrm{~m}$ pour les parcelles résidentielles, Godoy justifia dans un autre article qu'une devanture de $12 \mathrm{~m}$ correspondait à celle des cités ouvrières aux Etats-Unis, sachant que la norme commune là-bas était de $18 \mathrm{~m}$ (Godoy 1932: 22).

L'administration municipale standardisa les projets de lotissement au cours des années trente. Jusque-là, comme les pouvoirs publics prêtaient surtout attention à la création de voirie, les projets de lotissement dépendaient de la législation sur l'alignement, d'où la confection de plan d'alignement au moment de leur approbation (Figure 5). Parfois, ces plans indiquaient les divisions des îlots en parcelles et/ou la numérotation de ces mêmes îlots et des parcelles, ce qui rapprochait le plan d'un catalogue de parcelles. Il aura fallu attendre le Code Godoy pour que surgissent les premiers PAL tels que nous les connaissons aujourd'hui: des plans sur lesquels figurent, en rouge, les rues, îlots et parcelles projetés, toujours numérotés et mesurés, et en noir le cadastre de la ville (Figure 7 et 8). Pour individualiser les PAL, l'administration opta pour un système de numéros cerclés deux fois de rouge, au coin supérieur droit des feuilles, quand elle ne cerclait le numéro des PAA qu'une seule fois. Tout comme les PAA, la SMU se charge d'archiver les PAL. Du point de vue graphique, les PAL se révèlent avant tout une sophistication de la technologie déjà présente dans les projets de lotissement: comme ils impliquent la création de voirie, l'administration les considère également comme des plans d'alignement et leur attribue un numéro cerclé une fois de rouge. Autrement dit, les projets approuvés de lotissement sont en même temps PAL et PAA, comme on peut le voir sur les figures 2, 7 et 8. D’un autre côté, les projets de démembrement (cf. note 2) reçoivent seulement un numéro de PAL. La distinction entre démembrement et lotissement trouve donc une traduction graphique simple dans le jeu des numérotations. Il faut également relever cette connexion importante dans le cadre de l'infrastructure documentaire de l'administration municipale: l'obligation, établie dans le Code de la Construction de 1937 (Décret n.6.00o du premier juillet 1937), de joindre à la demande de permis de construire sur une certaine parcelle une copie de projet approuvé de lotissement (article 116). 
Figure 7: PAA 2.142 (PAA 2.525). Projet de lotissement dans le quartier de Madureira, Zone Nord de Rio de Janeiro, approuvé en 1936.

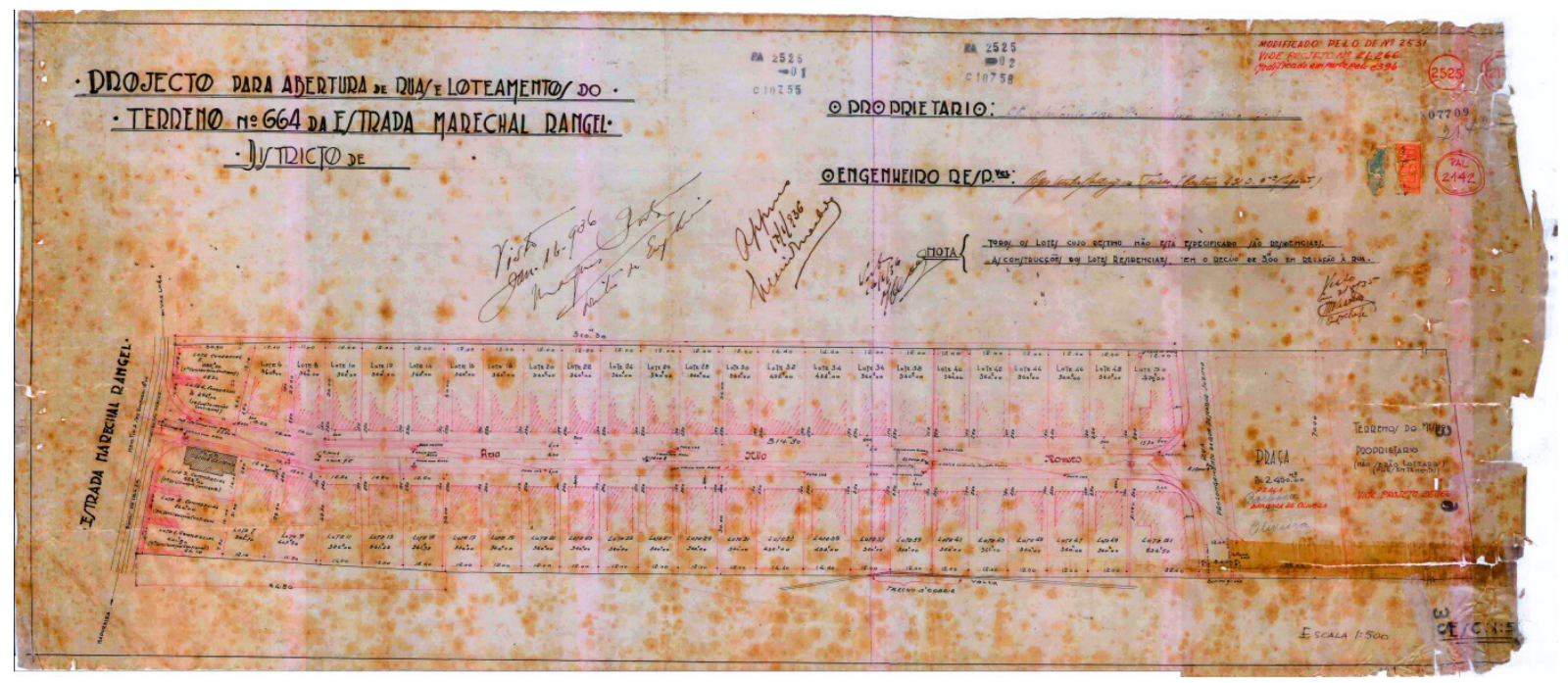

Source: SMU, PCRJ.

Figure 8 - PAL 48.584 (PAA 12.481). Projet de lotissement dans le quartier de Santíssimo, Zone Ouest de Rio, approuvé en 2014.

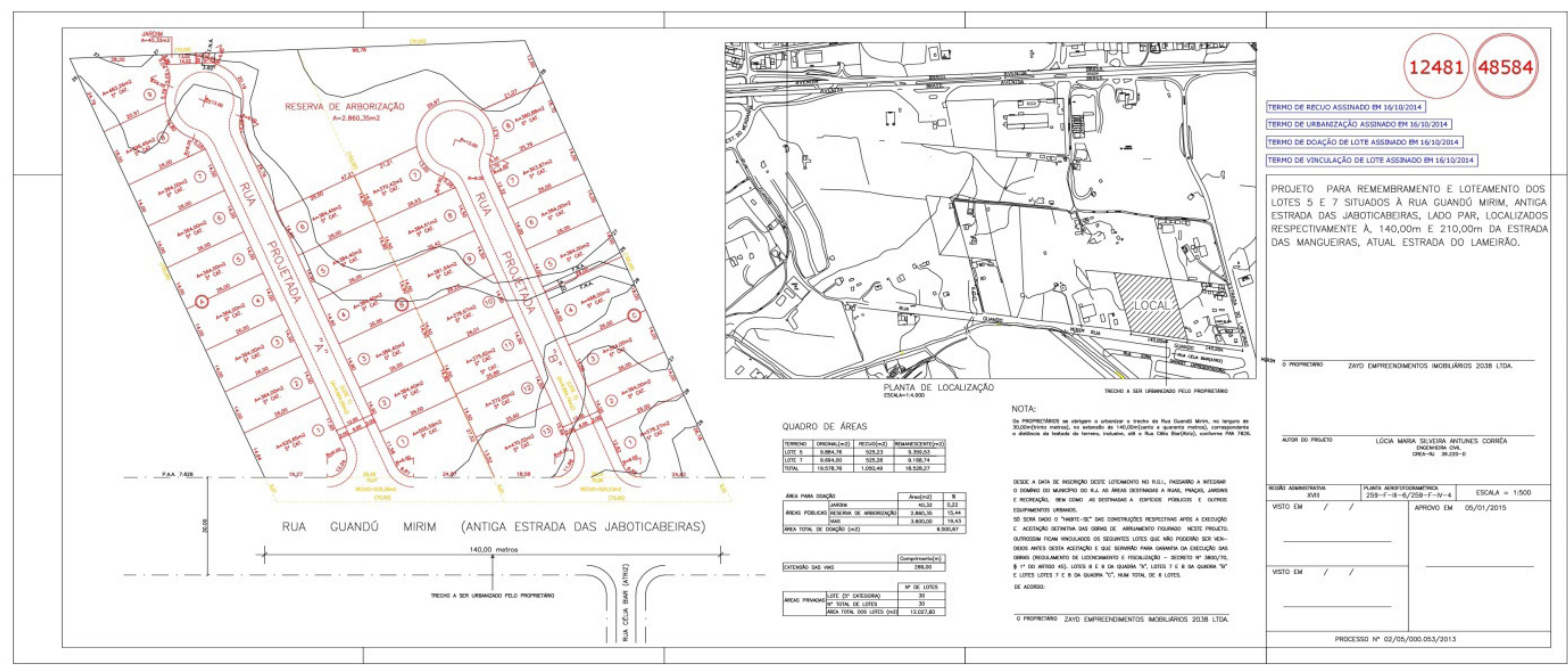

Source: SMU, PCRJ.

Enfin, nous signalerons que les PAL jouent un rôle très important en tant qu' " artefacts commerciaux ", au-delà de leur fonction " d'artefacts urbanistiques ». En effet, le Code Godoy reçut de nombreuses critiques des compagnies immobilières dans les années trente (Godoy 1932), qui contestaient jusqu’à la légalité du décret vu que, en matière de droit foncier, la prérogative est de la Fédération et non de la municipalité (à l'inverse, en matière d'urbanisme, la prérogative est de la municipalité, d'où l'imbroglio juridique). Le Décret-Loi n. 58 du 10 décembre 1937, qui « disposait sur le lotissement et la vente de terrain pour paiement en prestations ", issue du gouvernement central, vint résoudre cette ambiguïté juridique. Le décret visait principalement à garantir la sécurité juridique des personnes qui achetaient leurs parcelles à terme, une modalité alors en pleine expansion: la partie du Code Civil qui règlementait les transactions commerciales 
n'assurait pas la pleine propriété de la terre à qui payait à terme, du moins pas tant que n'avait été émis le registre définitif de l'achat, une fois réglées toutes les mensualités. Le décret prétendait aussi lutter contre l'usurpation (grilagem), c'est-à-dire contre la vente de terrains par des gens qui n'en étaient pas les vrais propriétaires, compromettant l'accès des acheteurs à la pleine propriété. C'est pourquoi il exigeait des lotisseurs qu'ils déposassent chez le notaire, avant même de commercialiser ou de faire la publicité des parcelles, un mémorial incluant " la relation chronologique des titres de propriété depuis 30 ans ", " un exemplaire du carnet ou du contrat-type d'engagement de vente des parcelles ", un " certificat négatif d'impôt » et enfin un projet de lotissement avec le dessin de l'immeuble, celui-ci servant à situer les terrains mis en vente. Quoiqu'il s'agisse de deux sphères distinctes, un même artefact, le projet de lotissement, fonctionnait en même temps comme outil de régulation urbanistique et comme instrument de régulation commerciale et foncière. Cette jonction nous semble renvoyer au rôle de la " raison graphique " dans l'organisation des rapports de propriété: avant même que n'existent les PAL, les lotisseurs recourraient aux catalogues de terrains pour vendre leurs parcelles.

\section{Conclusion: la périphérie et l'arquéologie de l'aménagement urbain}

En se promenant dans les lointains quartiers de la Zone Ouest carioca, souvent appelés « villas » ou " jardins ", on pourra contempler des rues qui, au lieu d’un nom, possède un numéro; de maisons qui, au lieu du numéro de la rue, utilisent pour adresse les numéros de la parcelle et de l'îlot où elles se situent ; des avenues droites et allongées qui parfois se perdent au milieu de nulle part, recouvertes par la végétation. Cette expérience particulière de l'espace provient de la technologie du lotissement, surtout dans ses aspects graphiques: si les rues, îlots et parcelles possèdent un numéro, c'est parce qu'elles appartiennent tout autant à un dessin, appelé " projet » ou " plan ", avant même d'entrer dans l'espace physique ; si les avenues sont droites et ne s'embarrassent d'aucun obstacle humain ou naturel, c'est parce qu'elles sont d'abord des lignes droites sur un dessin. À la base des lotissements périphériques, il y a donc une " raison graphique ", une façon d'ordonner le réel (dans ce cas, l'espace physique de la ville) à partir de figures disposées sur une superficie visible et facilement manipulable. Toutefois, cette technologie graphique ne débouche sur aucune vision " unifiée et simplifiée " de la ville comme le suggèrent parfois les théoriciens du plan orthogonal ; plutôt que de rendre la société « lisible », les artefacts graphiques résolvent des problèmes pratiques de gouvernement. En particulier, la technologie du lotissement, telle que nous la connaissons, est née de la préoccupation croissante pour la prévision, la planification des besoins collectifs: pour garantir de bonnes conditions hygiéniques, esthétiques et morales aux habitants (pauvres) des nouveaux quartiers ; pour que le marché immobilier fonctionne correctement, sans porter préjudice aux acheteurs, il fallait intervenir jusque dans la forme des parcelles, de façon à accorder tous les éléments, humains et matériels, entre eux. Les normes techniques, telles que la standardisation des parcelles, ne sont jamais anodines, elles contiennent de précieuses informations sur la façon dont le gouvernement problématise la dimension physique de l'espace urbain.

La méthode généalogique que nous avons adoptée ici nous a montré que la périphérie n’est pas extérieure à la ville, « formelle » ou non, bien au contraire: à mesure que la ville s’étendait, de nouveaux problèmes politiques surgissaient, de nouvelles technologies juridico-administratives également, qui se sont appliquées d'abord à la frontière urbaine. Si dans le centre de la ville, la " régularisation » de la trame viaire s'avérait une tâche dispendieuse, en périphérie le gouvernement pouvait imposer de nouvelles normes bien plus rigides - là en périphérie, la ville pouvait se développer en accord avec le modèle rêvé des élites occidentales. De même avec la formation des parcelles: si en centre-ville le remembrement se révélait une opération particulièrement complexe, en périphérie il était possible d’imposer le modèle de la 
cité-jardin et de résoudre ainsi le problème du logement. Parallèlement, la périphérie a posé de nouveaux problèmes au gouvernement de la ville, ce qui a amené une sophistication des instruments. L'ouverture simultanée de plusieurs rues, à l'initiative souvent de grandes compagnies capitalistes, ou la vente de parcelles à terme contraignirent le gouvernement à proposer de nouveaux outils, tels que les projets de lotissement. Loin d'une « ville illégale », un véritable laboratoire de l'aménagement urbain. Néanmoins, comme dans n'importe quel laboratoire, toutes les expériences ne sont pas forcément couronnées de succès. Si le gouvernement a beaucoup incité les compagnies à lotir la périphérie au siècle dernier, pour donner aux pauvres accès à la propriété, ces incitations n’ont pas nécessairement produit le genre de logement espéré par le gouvernement: les innombrables défaillances des infrastructures urbaines en périphérie le prouvent constamment. Mais cela est déjà une autre histoire.

Reçu le 31 décembre 2016 ; Accepté le 23 octobre 2017

\section{Bibliographie}

ABREU, Maurício de Almeida. 2013. Evolução urbana do Rio de Janeiro. Rio de Janeiro: Instituto Pereira Passos. ANDREATTA, Verena. 2006. Cidades quadradas, paraísos circulares. Rio de Janeiro: Mauad X. A NOITE. 1923. O problema de melhoramentos das cidades. A Noite, Rio de Janeiro, 3 out. 1923, p. 3. AZEVEDO, André Nunes de. 2013. "A cura pela técnica: o Clube de Engenharia e a questão urbana na cidade do Rio de Janeiro na virada do século XIX ao XX”. Locus: Revista de História, Juiz de Fora, 19(2): 273-292. BONDUKI, Nabil. 1999. Origens da habitação social no Brasil. São Paulo: Estação Liberdade/ FAPESP. ANAIS DA BIBLIOTECA NACIONAL. 1962. Rio de Janeiro: Biblioteca Nacional, 82. ARCHIVO DO DISTRICTO FEDERAL. 1894. Rio de Janeiro: Archivo Municipal, 1. COMMISSÃO DE MELHORAMENTOS DA CIDADE DO RIO DE JANEIRO (CMCRJ). 1875. Primeiro Relatório da Commissão de Melhoramentos da Cidade do Rio de Janeiro. Rio de Janeiro: Typographia Nacional.

.1876. Segundo Relatório da Commissão de Melhoramentos da Cidade do Rio de Janeiro. Rio de Janeiro:

Typographia Nacional.

CONNIFF, Michael L. 2006. Política urbana no Brasil. Rio de Janeiro: Relume Dumará.

CORBIN, Alain. 1982. Le miasme et la jonquille. Paris: Flammarion.

CORTADO, Thomas. 2018. À beira da cidade: política e poética da urbanização no Jardim Maravilha, "loteamento proletário" da Zona Oeste carioca. Thèse de doctorat, Université Fédérale de Rio de Janeiro (UFRJ), Musée National (MN), Programme de Troisième Cycle en Anthropologie Sociale (PPGAS) - Rio de Janeiro. Disponible sur: https://www.academia.edu/36401995/\%C3\%8o_beira_da_cidade_po\%C3\%Agtica_e_ pol\% $\mathrm{C}_{3} \% \mathrm{ADtica}$ _da_urbaniza\% $\mathrm{C}_{3} \% \mathrm{~A} 7 \% \mathrm{C}_{3} \% \mathrm{~A}_{3}$ __no_Jardim_Maravilha_loteamento_prolet\% $\mathrm{C}_{3} \%$ Arrio_ da_Zona_Oeste_carioca. Accessible le: 22 avril 2018.

COSTA, Jurandir Freire. 1979. Ordem médica e norma familiar. Rio de Janeiro: Edições Graal. CERTEAU, Michel de. 199o. L'invention du quotidien I: Arts de faire. Paris: Gallimard. FOUCAULT, Michel. 2004. Sécurité, territoire, population. Paris: Gallimard/ Seuil. FOURCAULT, Annie. 200o. La banlieue en morceaux. Grâne: Créaphis.

GAGLIARDO, Vinicius Cranek. 2014. "A intendência de polícia e a civilização do Rio de Janeiro oitocentista". URBANA, 6(8). Disponível em: <http://www.ifch.unicamp.br/ojs/index.php/urbana/issue/view/65/ showToc>. Acesso em: 13 de nov. 2016. 
GODOY, Armando Augusto de. 1932. "A improcedência de algumas objeções contra a lei sobre a abertura de ruas e loteamentos de terrenos". Revista da Directoria de Engenharia, 1(1): 21-27.

. 1935. "As consequências econômicas e sociais dos princípios do urbanismo". Revista do Clube de

Engenharia, 2(14): 711-722.

. 1943. A URBS e os seus problemas. Rio de Janeiro: Jornal do Commercio.

GOODY, Jack. 1979. La raison graphique. Paris: Les Éditions de Minuit.

HAROUEL, Jean-Louis. 1977. "Les fonctions de l'alignement dans l'organisme urbain”. Dix-Huitième Siècle, 9: 135-149.

HULL, Matthew S. 2012. Government of paper. Berkeley/ Los Angeles/ Londres: University of California Press. LATOUR, Bruno. 1987. Science in action. Cambridge, Massachusetts: Harvard University Press.

.; CALLON, Michel. 2015. "Unscrewing the big Leviathan: how actors macrostructure reality and how sociologists help them to do so". In: K. D. Knorr-Cetina; A.V. Cicourel (orgs.), Advances in social theory and methodology. Londres/ Nova Iorque: Routledge. pp. 277-303.

LEFEBVRE, Henri. 1999. La production de l'espace. Paris: Anthropos.

MACHADO, Roberto et al. 1978. Danação da norma. Rio de Janeiro: Graal.

MARICATO, Ermínia (Org.) 1979. A produção capitalista da casa (e da cidade) no Brasil industrial. São Paulo:

Alfa-Omega.

MILLER, Peter; ROSE, Nikolas. 1990. “Governing economic life”. Economy and Society, 19(1):1-31.

MITCHELL, Timothy. 1991. Colonising Egypt. Berkeley/ Los Angeles/ Londres: University of California Press.

PEIXOTO, Domingos Ribeiro dos Guimarães. 2008. A saúde pública no Rio de Dom João. Rio de Janeiro: Editora Senac Rio. pp. 84-118.

PEREIRA, Magnus Roberto de Mello. 2001. "Almuthasib - Considerações sobre o direito de almotaçaria nas cidades de Portugal e suas colônias". Revista Brasileira de História, 21(42): 365-395.

PEREIRA, Margareth da Silva. 2001. "As palavras e a cidade: o vocabulário da segregação em São Paulo". Espaço e Debates, 42: 31-47.

PINTO, Sandra M. G. 2016. “A regulação jurídica das fachadas em Portugal (séc. XIV-XIX)”. Revista de Estúdios Histórico-Jurídicos, 38: 149-177.

PINTO, Sandra M. G. 2012. As interações no sistema das operações urbanísticas nos espaços urbanos portugueses até meados de oitocentos. Thèse de doctorat, Université de Coimbra, Faculté de Sciences et Technologies Coimbra.

PREFEITURA DO DISTRITO FEDERAL (PDF). 1894. Código de posturas. Rio de Janeiro: Papelaria e Typographia Mont'Alverne.

. 1930. Cidade do Rio de Janeiro: extensão - remodelação - embellezamento. Paris: Foyer Brésilien.

REIS FILHO, Nestor Goulart. 200o. Quadro da arquitetura no Brasil. São Paulo: Editora Perspectiva.

REIS, José de Oliveira. 1977. O Rio de Janeiro e seus prefeitos: projetos de alinhamento. Rio de Janeiro: Prefeitura da Cidade do Rio de Janeiro.

RIBEIRO, Luiz Cesar de Queiroz. 1997. Dos cortiços aos condomínios fechados: as formas de produção da moradia na cidade do Rio de Janeiro. Rio de Janeiro: Civilização Brasileira.

ROLNIK, Raquel. 2005. A cidade e a lei: legislação, política urbana e territórios na cidade de São Paulo. São Paulo: FAPESP/Studio Nobel.

ROSE-REDWOOD, Reuben Skye. 2008. "Genealogies of the grid: revisiting Stanislawski's search for the origin of the grid-pattern town". The Geographical Review, 98(1): 42-58.

SAMPAIO, Maria Ruth Amaral de; LIRA, José Tavares Correia de Lira. 2010. "Loteamento". In: C. Topalov et al. (orgs.), Les aventures des mots de la ville. Paris: Robert Laffont. pp. 670-677. 
SANTOS, Carlos Nelson Ferreira dos. 1977. "Volviendo a pensar en 'favelas' a causa de las periferias". Nueva Sociedad, 30: 22-38.

. 1980. "Velhas novidades nos modos de urbanização brasileiros". In: L. d. P. Valladares (org.),

Habitação em questão. Rio de Janeiro: Zahar. pp. 17-48.

SCOTT, James Campbell. 1998. Seeing like a State. New Haven, London: Yale University Press.

SILVA, Lúcia. 2003. História do urbanismo no Rio de Janeiro. Rio de Janeiro: E-Papers Serviços Editoriais.

VALLADARES, Lícia do Prado; FIGUEIREDO, Ademir. 1981. "Habitação no Brasil”. BIB - Boletim Informativo e Bibliográfico de Ciências Sociais, 11: 25-49.

VERÍSSIMO, Antônio Augusto. 2005. Parcelamento do solo na cidade do Rio de Janeiro: um estudo sobre a produção informal da década de 40 aos anos go. Mémoire de master, Université Fédérale de Rio de Janeiro (UFRJ), Institut de Recherches en Aménagement Urbain et Régional (IPPUR) - Rio de Janeiro.

\section{Thomas Cortado}

Université Fédérale de Rio de Janeiro, Musée National, Programme de Troisième Cycle en Anthropologie sociale (PPGAS), Rio de Janeiro, Brésil.

Courriel de l'auteur: cortado.thomas@gmail.com 